\title{
Effects of Transmission Ratio on the Nonlinear Vibration Characteristics of a Gear-Driven High-Speed Centrifugal Pump
}

\author{
Anmin Zhou, ${ }^{1}$ Lulu Zhai $\mathbb{D}^{1,2}{ }^{1,2}$ Zuchao Zhu, $^{1}$ Jia Guo, ${ }^{3}$ Xinglin Zhang, $^{2}$ and Baoling Cui ${ }^{1}$ \\ ${ }^{1}$ State-Province Joint Engineering Lab of Fluid Transmission System Technology, Zhejiang Sci-Tech University, \\ 928\# No. 2 Avenue, Xiasha, Hangzhou, China \\ ${ }^{2}$ Hefei General Machinery Research Institute Co., Ltd., Hefei 230031, China \\ ${ }^{3}$ Zhejiang Institute of Mechanical \& Electrical Engineering Co., Ltd., Hangzhou, China \\ Correspondence should be addressed to Lulu Zhai; zhailulu@zstu.edu.cn
}

Received 2 November 2020; Revised 17 December 2020; Accepted 21 December 2020; Published 8 January 2021

Academic Editor: Ling Zhou; lingzhou@ujs.edu.cn

Copyright (C) 2021 Anmin Zhou et al. This is an open access article distributed under the Creative Commons Attribution License, which permits unrestricted use, distribution, and reproduction in any medium, provided the original work is properly cited.

\begin{abstract}
The gear transmission system is widely used in high-speed centrifugal pump to improve the operating speed and hydraulic performances of the whole pump. Vibration characteristics and the stability of these high-speed rotor systems with gear transmission have great impacts on the stability of the whole fluid transmission system of the plant. Based on the lumped-mass method and the principle of displacement equilibrium of the rotor system, a coupled lateral-torsional dynamic model describing the gear-rotor-seal-bearing (GRSB) system of high-speed centrifugal pumps which has considered the nonlinear factors within the gear pair, nonlinear forces of bearings, and those of the seals is proposed. Then, the stability and nonlinear vibration responses of a model GRSB system under different gear transmission ratios $(i)$ have been studied. The following conclusions are drawn from the results: (1) The components with frequencies like $f_{p}, f_{g}, f_{m}$, and $2 f_{m}$ have great impacts on the vibration responses of the gear pair, especially the $f_{m}$ component; moreover, the amplitude of $f_{m}$ first increases and then decreases with the ratio increase and reaches the maximum value under the ratio of 3. (2) A jump motion state will occur when the ratio $i$ is 1.25 and the stability of the system is obviously worse than the bifurcation state. Quite different from those under the other states, under this jump motion state, the $0.2 f_{g}$ component and $0.5 f_{p}$ component will appear in the vibration responses of both gears and become the most contributed two factors to the responses of the driven gear. (3) In the design process, the transmission ratio of a high-speed centrifugal pump with a simplified GRSB system should be specially designed to avoid the jump-point state and the maximum-amplitude-of- $f_{m}$ state to ensure the stability of the system as well as reduce the mechanical impacts and noises.
\end{abstract}

\section{Introduction}

High-speed centrifugal pumps which are characterized by high speed and low specific speed are widely used as the key equipment of fluid transport in petrochemical and nuclear energy fields [1]. The vibration characteristic analysis is a key problem in the design and optimization of this kind of pump and is also the key to ensuring the stable operation of the pump. Generally, the rotor system of the high-speed centrifugal pump includes gears, seals, bearings, and rotating shaft; therefore, the system can be simplified to a gear-rotorseal-bearing (GRSB) system. Nonlinear factors, including bearing forces, seal forces, and meshing forces of gears acting on the rotor system of these high-speed centrifugal pumps, make the system show significant nonlinear vibration characteristics. Previous studies showed that the nonlinear bearing force, seal force, and inner flow directly induced the instability problem in these pumps [2-4]. Beyond that, the interactions of lateral vibration and torsional vibration make the dynamic behaviors of the GRSB system more complex and difficult to predict [5]. Therefore, the vibration analyses of these GRSB systems should be undertaken by combining the studies of bearings, seals, gear pairs, and coupled lateraltorsional vibration analyses.

As main supporting elements, rolling bearings are widely used in centrifugal pump for the compact structure, low friction resistance, and high mechanical efficiency. El-Sayed et al. [6] proposed the method for calculating the bearing 
stiffness using Hertz's contact theory and analyzed the deflections of the outer and inner rings caused by an applied load. Harsha et al. [7-10] developed a dynamic model of a bearing-rotor system based on the Lagrange equation and examined the influences of the pretightening force, ball number, bearing clearance, and wavy degree on the stability of the system. Kankar et al. [11] derived a mathematical formulation with consideration of the nonlinear springs and nonlinear damping at the contact points of rolling elements and races and investigated the effects of bearing running surface waviness on the nonlinear vibrations of the rotorbearing system. Wang et al. [12] developed an analytical model to investigate the effects of inhomogeneity in races on the dynamic behaviors of rolling bearing. The results showed that the inhomogeneity in the inner race had greater influences than that in the outer race. Yang et al. [13] analyzed the varying compliance resonance characteristics in a ballbearing-rotor system and compared the vibration responses of the system in a large speed range with the consideration of different ball numbers and different rotor eccentricities.

In addition to the investigations of rolling bearings and rotor-bearing system, the characteristics of annular seals, vibration performances, and stability of rotor-bearing-seal systems are also research hotspots in recent decades. Muszynska [14] introduced a fluid velocity ratio to describe the whirling motion of the seal and proposed a model for the rotor-bearing-seal system, which is proved to be of great significance to the stability analysis of the rotor system by experiments. Leung et al. [15] studied the Hopf bifurcation behavior of a rotor-seal system under the unbalanced rotor mass based on the Muszynska nonlinear seal force model. Cheng et al. [16] investigated the nonlinear behaviors of a rotor-bearing-seal system based on Muszynska's nonlinear seal fluid dynamic force model. The simulation results indicated that the dynamic behavior of the system depends on the rotational speed, seal clearance, and pressure difference of the seals. Li et al. [17] established a new dynamic model of a rotor system based on the Hamilton principle and finite element method (FEM) and compared the dynamic behaviors of the system with different seal pressure differences and mass eccentricity. Peng et al. [18] developed an excitation method based on the Bently-Muszynska model and discussed the optimum shape of the balancing drum to reduce the excessive vibration under high rotational speed operating conditions. Zhang et al. [19] derived the motion equations of a rotor-bearing-labyrinth-seal system and analyzed effects of the inlet pressure and seal length on the stability of the system by spectrum cascades, bifurcation diagrams, and Poincare maps. Zhou et al. [20] explored nonlinear characteristics of the system under different seal clearances, seal lengths, and seal radiuses based on the nonlinear dynamic model and optimized the stability of the rotor system through a genetic algorithm. Later, Zhou [21] proposed a dynamic vibration model for the pump-turbine rotor system and studied the nonlinear dynamic characteristics of crown seal force and stability of the rotor system with different seal channel clearance and air gap. Wang et al. [22] developed a coupled dynamic model of multistage pump rotor system based on FEM, whose maximum errors were $5.5 \%$ compared to the experimental results. Recently, Zhou et al. [23] proposed a novel axial dynamic model considering transient forces of balancing drum seal and analyzed the inner chamber pressure changes with the increase of the axial gap and rotational speed. The results showed that the inner chamber pressure was more sensitive to the inlet pressure than the rotational speed. Bai et al. [24] tested the vibration characteristics of a cantilever multistage centrifugal pump under different flow rates and valued its operating stability. The results showed that the vibration of the unbalanced mass of the rotor was the most important factor that induced excessive vibration.

As the gear transmission system and the interactions between the lateral vibration and the torsional vibration have a great influence on the stability and vibration characteristics of the rotor system, researches on relevant topics have been conducted in recent years. Kaharman et al. [25] examined the nonlinear frequency response characteristics of a spur gear pair with backlash under external and internal excitation by harmonic balance method and built a three-degree-of-freedom dynamic model for a gearrotor-bearing system [26]. Besides, the influences of nonlinear modal interactions, internal static transmission error excitations, and external torque excitations were discussed. Rao et al. [27] studied the lateral response of the gear-rotor system due to torsional excitation and the results showed that the coupling mechanism may be harmful to the operation of geared turbine rotors. Stephanos et al. [28] investigated the response and stability characteristics of the gear-rotor system with external loads and gear meshing loads. Vedmar et al. [29] analytically described the dynamic gear tooth forces and bearing forces and found that the dominant vibration mode for the gear contact is the one where the gear tooth deformation appears. Lee et al. [30] presented a calculation method for the unbalance response orbit of a gear-coupled two-shaft rotor-bearing system and observed bumps in the unbalance responses at the first torsional natural frequency because of the coupling between the lateral and torsional dynamics due to gear meshing. Anoshirvan et al. [31] formulated a generalized nonlinear time-varying (NLTV) dynamic model of a spur gear pair, where the backlash, external excitation, and static transmission error are included, using Melnikov analysis. The global homoclinic bifurcation and the transition to chaotic behavior of a nonlinear gear system were studied based on the proposed model. Li et al. [32] established a lumped-parameter nonlinear dynamic model of a helicalgear-rotor-bearing system and obtained vibration response of this system with different rotational speed and eccentricity. Habibollah et al. [33] investigated the effects of tooth profile modification on the nonlinear dynamic behaviors of the straight-bevel-gear systems and employed the optimum tooth profile to modify the dynamic response of the bevel gear through a genetic algorithm. Jerzy et al. [34] proposed a model describing the gear backlash which involved polynomial functions of the third degree and the logarithmic equation and investigated the numerical mapping characteristics of the gear backlash. Tang et al. [35] discussed the fault diagnosis performances of gears and 
bearings in rotating machinery based on different CNN methods.

It can be concluded that, in the previous studies, the coupled lateral-torsional vibration dynamics analysis of the system for high-speed centrifugal pump, especially the research of gear parameters on the stability and vibration characteristics of the high-speed pump rotor system, is relatively rare. Thus, in this paper, a coupled lateral-torsional vibration dynamic model considering the nonlinear bearing forces, seal forces, and dynamic meshing forces of the gears and the forth-order-Runge-Kutta-based solution for this model are established. And the effects of the transmission ratio on the nonlinear vibration characteristics of the model GRSB system are investigated based on the proposed model.

\section{Dynamic Model of a Gear-Rotor-Seal-Bearing (GRSB) System}

Figure 1 shows the $3 \mathrm{D}$ structure diagram of the rotor system of a high-speed centrifugal pump. The system is composed of driving gear, driven gear, rolling bearings, rotor, seal, and elastic rotating shafts. The forces and torque acting on the system are shown in Figure 2, including input and output torque, meshing force, and nonlinear seal force and bearing force. According to the lumped-mass method, the rolling bearings, driving gear, driven gear, and rotor can be simplified as 7 mass nodes represented by $o_{j}(j=b 1, b 2, b 3, b 4, p$, $g, r, d)$, whose equivalent mass is $m_{b 1}, m_{b 2}, m_{b 3}, m_{b 4}, m_{p}, m_{g}$, and $m_{r}$, respectively. The driving torque of the rotor system acts on node $d . x_{j}$ and $y_{j}(j=b 1, b 2, b 3, b 4, p, g, r)$ represent the displacements of $o_{j} j=b 1, b 2, b 3, b 4, p, g, r, d$ in $x$ and $y$ directions.

$k_{m}(t)$ and $c_{m}(t)$ are time-varying meshing stiffness and damping of the gear pair; $\delta(t)$ is the internal excitation error in the meshing process of gears; $\omega_{1}$ and $\omega_{2}$ denote the angular velocity of the driving and driven shafts, respectively; $\varphi_{j}(j=d, p, g, r)$ refers to the angular displacements of $o_{j}(j=d, p, g, r)$; and $\varphi_{d}$ refers to the angular displacement of the node acted by input loads. The angular displacements can be expressed as

$$
\begin{aligned}
& \phi_{d}=\omega_{1} t+\theta_{d}, \\
& \phi_{p}=\omega_{1} t+\theta_{p}, \\
& \phi_{g}=\omega_{2} t+\theta_{g}, \\
& \phi_{r}=\omega_{2} t+\theta_{r},
\end{aligned}
$$

where $\theta_{j}(j=d, p, g, r)$ are the torsional vibration displacements of the nodes mentioned above.

2.1. Nonlinear Dynamic Meshing Force of Gears. As the nonlinear forces due to gear meshing have great influences on vibration characteristics of the rotor system, factors that will affect the nonlinear forces are considered in the proposed dynamic model of this paper, including tooth surface friction, mass eccentricity, and gear backlash in the gear pair.

Figure 3 illustrates the forces and displacements within the gear system the gear pair of the GRSB system. $e_{p}$ and

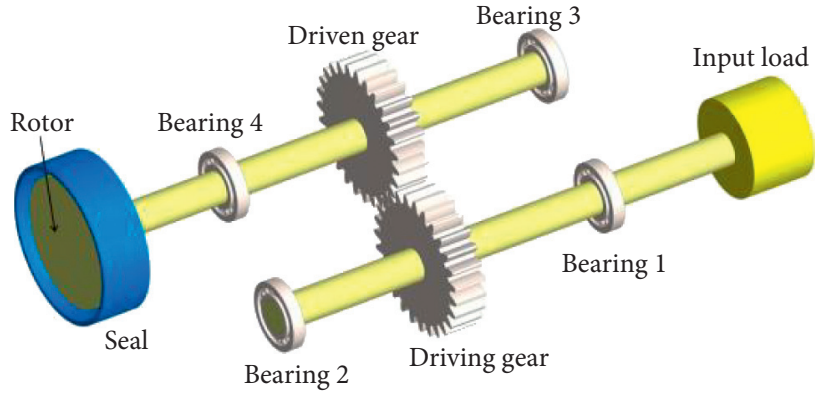

Figure 1: Gear-rotor-seal-bearing (GRSB) system.

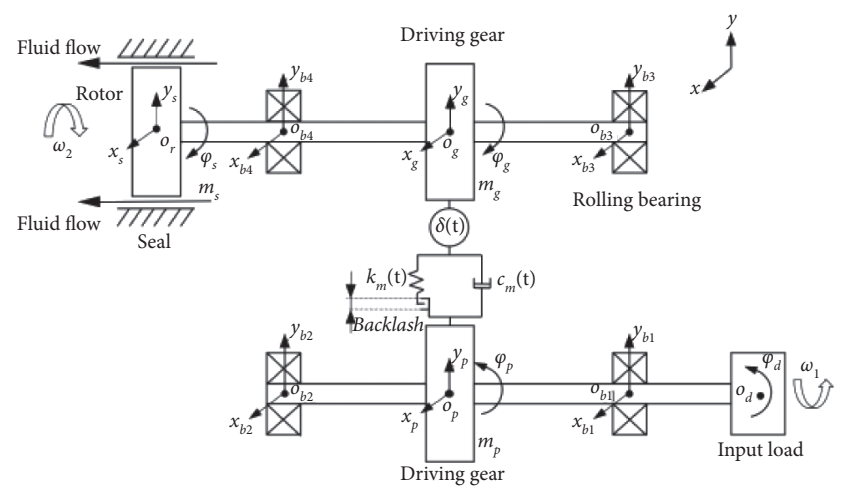

FIgURE 2: Schematic diagram of structure and forces of GRSB system.

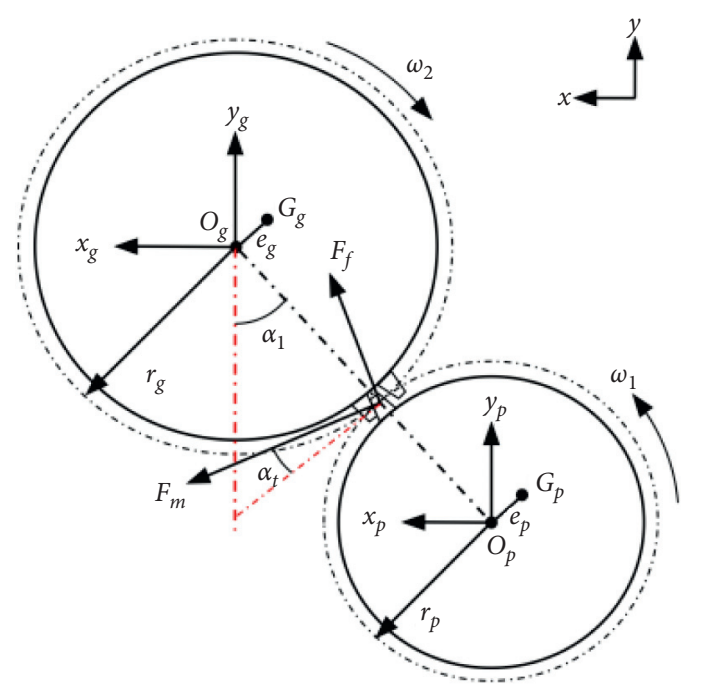

Figure 3: Forces and displacements within the gear system.

$e_{g}$ are the mass eccentricity on the driving and driven gear; $G_{p}$ and $G_{g}$ are the barycenters of the driving and driven gear; $r_{p}$ and $r_{g}$ are the pitch radii of the driving and driven gears, respectively; $\alpha_{1}$ is the angle between the center line of the gears and the vertical direction; $\alpha_{t}$ is the pressure angle of the gear; $F_{m}$ and $F_{f}$ are the dynamic meshing force and friction force of gear. Referring to the elastic theory, $F_{m}$ can be expressed as follows: 


$$
F_{m}=k_{m}(t) \cdot f(S)+c_{m}(t) \cdot \mathrm{d} S,
$$

where $k_{m}$ and $c_{m}$ can be described as equation (3); $f(S)$ is the function of the backlash and can be expressed as equation (4) [36].

$$
\begin{aligned}
& k_{m}(t)=k_{\mathrm{av}}+k_{\mathrm{am}} \sin \left(\omega_{1} \cdot z_{p} \cdot t\right), \\
& c_{m}(t)=2 \xi_{m} \sqrt{\frac{k_{m}(t) r_{p}^{2} r_{g}^{2} I_{p} I_{g}}{r_{p}^{2} I_{p}+r_{g}^{2} I_{g}},} \\
& f(S)= \begin{cases}S-\frac{\text { backlsh }}{2}, & S>\frac{\text { backlsh }}{2}, \\
0, & -\frac{\text { backlsh }}{2} \leq S \leq \frac{\text { backlsh }}{2}, \\
S+\frac{\text { backlsh }}{2}, & S>\frac{\text { backlsh }}{2},\end{cases}
\end{aligned}
$$

where $k_{\mathrm{av}}$ is the mean of the stiffness; $k_{\mathrm{am}}$ represents the amplitude of the stiffness; $\zeta_{m}$ is the damping ratio of the gear.

$S$ represents the relative displacement produced by the bending-torsional coupled vibrations of the driving and driven gears. $S$ can be obtained by equation (5) [32].

$$
\begin{aligned}
S= & {\left[\left(x_{p}+e_{p} \cos \phi_{p}\right)-\left(x_{g}+e_{g} \cos \phi_{g}\right)\right] \cos \left(\alpha_{1}-\alpha_{t}\right) } \\
& +\left[\left(y_{p}+e_{p} \sin \phi_{p}\right)-\left(y_{g}+e_{g} \sin \phi_{g}\right)\right] \\
& \cdot \sin \left(\alpha_{1}-\alpha_{t}\right)+r_{p} \theta_{p}-r_{g} \theta_{g}-\delta(t),
\end{aligned}
$$

where $\delta(t)$ can be described as follows:

$$
\delta(t)=\delta_{\mathrm{av}}+\delta_{\mathrm{am}} \sin \left(\omega_{1} \cdot z_{p} \cdot t\right)
$$

$\delta_{\mathrm{av}}$ and $\delta_{\mathrm{am}}$ are the mean meshing error and fluctuation amplitude, respectively; $z_{p}$ and $z_{g}$ are the number of teeth of the driving and driven gear.

The meshing force between gear teeth is decomposed into the $x$ and $y$ directions, and it can be obtained as follows:

$$
\begin{aligned}
& F_{m x}=F_{m} \cdot \cos \left(\alpha_{1}-\alpha_{t}\right), \\
& F_{m y}=F_{m} \cdot \sin \left(\alpha_{1}-\alpha_{t}\right) .
\end{aligned}
$$

According to the force analysis of the gear meshing process, the friction force $F_{f}$ of gear can be approximately expressed as

$$
\begin{aligned}
F_{f} & =\lambda \cdot f_{\lambda} \cdot F_{m}, \\
F_{f x} & =F_{f} \sin \left(\alpha_{1}-\alpha_{t}\right), \\
F_{f y} & =F_{f} \cos \left(\alpha_{1}-\alpha_{t}\right),
\end{aligned}
$$

where $F_{f x}$ and $F_{f y}$ are the components of $F_{f}$ in the $x$ and $y$ directions, respectively; $f_{\lambda}$ is the equivalent friction coefficient; $\lambda$ is the direction coefficient of the gear tooth friction, which can be calculated by the following equations [37]:

$$
\begin{aligned}
\lambda_{p} & =\left(r_{p}+r_{g}\right) \tan \alpha_{t}-\sqrt{r_{p}^{2}-r_{g}^{2}}+r_{p} \omega_{1} t \\
\lambda_{g} & =\left(r_{p}+r_{g}\right) \tan \alpha_{t}-\lambda_{p} \\
\lambda & =\operatorname{sgn}\left[\dot{\theta}_{p} \lambda_{p}-\dot{\theta}_{g} \lambda_{g}\right] .
\end{aligned}
$$

2.2. Nonlinear Rolling Bearing Force. The schematic diagram of rolling bearing and the forces acting on it are shown in Figure 4 . $\mathrm{Nb}$ denotes the number of the rollers in the rolling bearing; $F_{j}$ is the force on the roller $j$; and the $F_{j}$ position $\beta_{j}$ is represented as

$$
\beta_{j}=\omega \cdot t+\frac{2 \pi(j-1)}{N b} .
$$

Disregarding the axial force on the bearing, the lateral vibration displacement at the journal position is assumed as $x$ and $y$. In accordance with Hertz contact theory, $F_{j}$ and the radial forces $F_{b x}$ and $F_{b y}$ received from the roller can be obtained using the following equations:

$$
\begin{aligned}
\Delta & =x \cos \beta_{j}+y \sin \beta_{j}, \\
F_{j} & =\mathrm{Kb} \Delta_{+}^{\frac{3}{2}} \\
F_{b x} & =\sum_{j}^{\mathrm{Nb}} F_{j} \cos \beta_{j} \\
F_{b y} & =\sum_{j}^{\mathrm{Nb}} F_{j} \sin \beta_{j},
\end{aligned}
$$

where $\Delta$ is the deformation of roller $j$ and $\mathrm{Kb}$ is a constant determined by the total deformation of the rolling body and the inner and the outer rings under the actions among them.

2.3. Nonlinear Seal Force and Torque Excitation. Muszynska's [13] nonlinear fluid force model within the seals is introduced in the dynamic model of the GRSB system. In this model, a velocity ratio $\gamma$ is proposed to describe the force-motion relations (shown in equation (12)) of the seal rotor and is used to solve the dynamic coefficients including stiffness $K_{\text {seal }}$ and damping $C_{\text {seal }}$ [38]. [14].

$$
\begin{aligned}
& -\left[\begin{array}{c}
F_{\text {seal } x} \\
F_{\text {seal } y}
\end{array}\right]=\left[\begin{array}{cc}
K_{\text {seal }}-M_{\text {seal }} \gamma^{2} \omega^{2} & \gamma \omega C_{\text {seal }} \\
-\gamma \omega C_{\text {seal }} & K_{\text {seal }}-M_{\text {seal }} \gamma^{2} \omega^{2}
\end{array}\right]\left[\begin{array}{l}
x_{r} \\
y_{r}
\end{array}\right] \\
& +\left[\begin{array}{cc}
C_{\text {seal }} & 2 M_{\text {seal }} \gamma \omega \\
M_{\text {seal }} \gamma \omega & C_{\text {seal }}
\end{array}\right]\left[\begin{array}{c}
\dot{x}_{r} \\
\dot{y}_{r}
\end{array}\right]+\left[\begin{array}{cc}
M_{\text {seal }} & 0 \\
0 & M_{\text {seal }}
\end{array}\right],
\end{aligned}
$$

where $F_{\text {sealx }}$ and $F_{\text {sealy }}$ are the fluid forces of the seal in the $x$ direction and $y$ direction, respectively.

The input torque $\left(T_{d}\right)$ acting on the GRSB system can be expressed by the following equation: 


$$
\begin{aligned}
T_{d} & =T_{\mathrm{av}}+T_{\mathrm{am}} \\
& =\frac{9550 P}{n_{1}}+0.1 \cdot T_{\mathrm{av}} \sin \left(\omega_{1} z_{1} t\right),
\end{aligned}
$$

where $T_{\mathrm{av}}$ is static torque and $T_{\mathrm{am}}$ is dynamic torque. Moreover, the torque acting on the driving gear $T_{p}$, the driven gear $T_{g}$, and the rotor $T_{r}$ can be simplified as

$$
\begin{aligned}
& T_{p}=T_{d} \cdot \eta, \\
& T_{g}=-T_{p} \cdot \frac{\eta}{i}, \\
& T_{s}=T_{g} \cdot \eta,
\end{aligned}
$$

where $\eta$ is transmission efficiency and $i$ is the transmission ratio of the gear pair.

2.4. Vibration Differential Equations of the GRSB System. In this paper, the dynamic equations of the model of GRSB system consider 18 degrees of freedom shown in equation (15).

$$
Z=\left[\theta_{d}, x_{b 1}, y_{b 1}, \theta_{p}, x_{p}, y_{p}, x_{b 2}, y_{b 2}, x_{b 3}, y_{b 3}, \theta_{g}, x_{g}, y_{g}, x_{b 4}, y_{b 4}, \theta_{r}, x_{r}, y_{r}\right]^{T}
$$

According to the principle of displacement equilibrium, the motion differential equations shown in equation (16) for the four bearings can be derived.

$$
\begin{aligned}
& m_{b 1} \ddot{x}_{b 1}+c_{s 1}\left(\dot{x}_{b 1}-\dot{x}_{p}\right)+k_{s 1}\left(x_{b 1}-x_{p}\right)=F_{b x 1}, \\
& m_{b 1} \ddot{y}_{b 1}+c_{s 1}\left(\dot{y}_{b 1}-\dot{y}_{p}\right)+k_{s 1}\left(y_{b 1}-y_{p}\right)=F_{b y 1}-m_{b 1} g, \\
& m_{b 1} \ddot{x}_{b 2}+c_{s 2}\left(\dot{x}_{b 2}-\dot{x}_{p}\right)+k_{s 2}\left(x_{b 2}-x_{p}\right)=F_{b x 2}, \\
& m_{b 1} \ddot{y}_{b 2}+c_{s 2}\left(\dot{y}_{b 2}-\dot{y}_{p}\right)+k_{s 2}\left(y_{b 2}-y_{p}\right)=F_{b y 2}-m_{b 2} g, \\
& m_{b 1} \ddot{x}_{b 3}+c_{s 3}\left(\dot{x}_{b 3}-\dot{x}_{g}\right)+k_{s 3}\left(x_{b 3}-x_{g}\right)=F_{b x 3}, \\
& m_{b 1} \ddot{y}_{b 3}+c_{s 3}\left(\dot{y}_{b 3}-\dot{y}_{g}\right)+k_{s 3}\left(y_{b 3}-y_{g}\right)=F_{b y 3}-m_{b 3} g, \\
& m_{b 1} \ddot{x}_{b 4}+c_{s 4}\left(\dot{x}_{b 4}-\dot{x}_{g}\right)+k_{s 4}\left(x_{b 4}-x_{g}\right)+c_{s 5}\left(\dot{x}_{b 4}-\dot{x}_{r}\right)+
\end{aligned}
$$

Similarly, the motion differential equations of the input load and driving gear can be expressed as

$$
\begin{aligned}
& I_{d} \ddot{\theta}_{d}+c_{t 1}\left(\dot{\theta}_{d}-\dot{\theta}_{p}\right)+k_{t 1}\left(\theta_{d}-\theta_{p}\right) \\
&= T_{d}, \\
& I_{p} \ddot{\theta}_{p} \quad+c_{t 1}\left(\dot{\theta}_{p}-\dot{\theta}_{d}\right)+k_{t 1}\left(\theta_{p}-\theta_{d}\right) \\
&=F_{m} r_{p}+T_{p}, \\
& m_{p} \ddot{x}_{p} \quad+c_{s 1}\left(\dot{x}_{p}-\dot{x}_{b 1}\right)+k_{s 1}\left(x_{p}-x_{b 1}\right) \\
&+c_{s 2}\left(\dot{x}_{p}-\dot{x}_{b 2}\right)+k_{s 2}\left(x_{p}-x_{b 2}\right) \\
&=F_{m x}+F_{f x}+m_{p} e_{p} \omega_{1}^{2} \cos \phi_{p}, \\
& m_{p} \ddot{y}_{p}+c_{s 1}\left(\dot{y}_{p}-\dot{y}_{b 1}\right)+k_{s 1}\left(y_{p}-y_{b 1}\right) \\
&+c_{s 2}\left(\dot{y}_{p}-\dot{y}_{b 2}\right)+k_{s 2}\left(y_{p}-y_{b 2}\right) \\
&= F_{m y}+F_{f y}-m_{p} g+m_{p} e_{p} \omega_{1}^{2} \sin \phi_{p} .
\end{aligned}
$$

The motion differential equations of the driven gear can be expressed as equation (18).
The motion differential equations of the rotor can be expressed as

$$
\begin{aligned}
& I_{r} \ddot{\theta}_{r}+c_{t 2}\left(\theta_{r}-\theta_{g}\right)+k_{t 2}\left(\theta_{r}-\theta_{g}\right)=T_{r} \\
& m_{r} \ddot{x}_{r}+c_{s 5}\left(\dot{x}_{r}-\dot{x}_{b 4}\right)+k_{s 5}\left(x_{r}-x_{b 4}\right) \\
& \quad=F_{\text {seal } x}+m_{r} e_{r} \omega_{2}^{2} \cos \phi_{r}, \\
& m_{r} \ddot{y}_{r}+c_{s 5}\left(\dot{y}_{r}-\dot{y}_{b 4}\right)+k_{s 5}\left(y_{r}-y_{b 4}\right) \\
& \quad=F_{\text {seal } y}-m_{r} g+m_{r} e_{r} \omega_{2}^{2} \sin \phi_{r} .
\end{aligned}
$$

$I_{j}(j=d, p, g, r)$ is the inertia moment of mass nodes $o_{d}, o_{p}$, $o_{g}$, and $o_{r}$, respectively. $k_{t 1}$ and $c_{t 1}$ are the torsional stiffness and damping of the driving shaft between nodes $o_{d}$ and $o_{p} ; k_{t 2}$ 


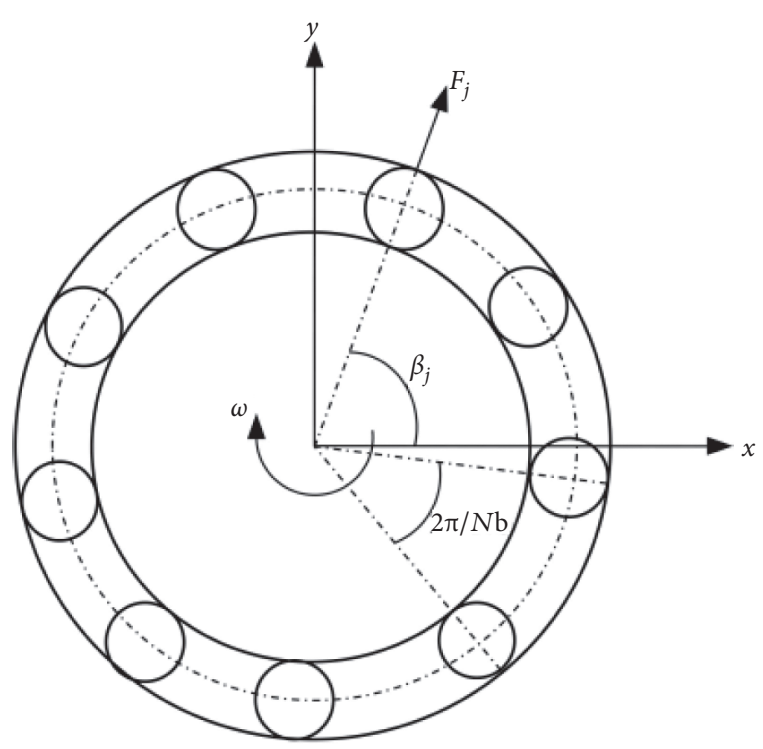

FIGURE 4: Schematic diagram of the rolling bearing system.

and $c_{t 2}$ are the torsional stiffness and damping of the driven shaft between nodes $o g$ and $o$; $k_{s 1}$ and $c_{s 1}$ are the bending stiffness and damping of the rotating shaft between node $o_{p}$ and node $o_{b 1} ; k_{s 2}$ and $c_{s 2}$ are the bending stiffness and damping of the rotating shaft between node $o_{p}$ and node $o_{b 2} ; k_{s 3}$ and $c_{s 3}$ are the bending stiffness and damping of the rotating shaft between node $o_{g}$ and node $o_{b 3} ; k_{s 4}$ and $c_{s 4}$ are the bending stiffness and damping of the rotating shaft between node $o_{g}$ and node $o_{b 4} ; k_{s 5}$ and $c_{s 5}$ are the bending stiffness and damping of the rotating shaft between node $o_{r}$ and node $o_{b 4}$.

Based on the proposed method above, the lateral-torsional coupled differential equation of a high-speed centrifugal pimp whose rotor system can be simplified as a GRSB system is established utilizing equations (16)-(19). Detailed parameters of the model GRSB system are provided in Table 1. And the nonlinear vibration response characteristics of the system can be obtained by solving the equations with the fourth-order Runge-Kutta method. The block diagram of the calculation program is illustrated in Figure 5. In this study, the displacement responses in $x$ direction of node $o_{r}$, which is the mass node of the rotor, are used to value the time-step independence. The calculation results with different time steps from $0.001 \mathrm{~s}$ to $0.00001 \mathrm{~s}$ are shown in Figure 6, and Figure 7 shows the amplitude of node $o_{r}$ in three crests with five different time steps. As is shown, when $\Delta t$ is less than $0.0001 \mathrm{~s}$, the responses curve changes little with the time step. Thus, to shorten the calculation time as well as ensure the calculation accuracy, the time step is set as $0.0001 \mathrm{~s}$. Besides, the integral time step is $\pi / 200$, and the convergence error is set as $1 \times 10^{-6}$.

\section{Vibration Responses Analysis of the GRSB System}

The transmission ratio has an important influence on the structure, layout, and overall design of the rotor system. And the influence of transmission ratio on the nonlinear
TABLE 1: Main parameters of the GRSB system.

\begin{tabular}{lc}
\hline Gear pair & \\
\hline$M$ & 3 \\
$Z_{g}$ & 17 \\
$m_{g}(\mathrm{~kg})$ & 1.115 \\
$I_{g}\left(\mathrm{~kg} \cdot \mathrm{m}^{2}\right)$ & $3.6 \times 10^{-4}$ \\
$k_{a}(\mathrm{~N} / \mathrm{m})$ & $4 \times 108$ \\
$k_{0}(\mathrm{~N} / \mathrm{m})$ & $2 \times 108$ \\
$e_{g}$ and $e_{p}(\mathrm{~m})$ & $1 \times 10^{-5}$ \\
$\delta_{m}$ and $\delta_{r}(\mathrm{~m})$ & $1 \times 10^{-5}$ \\
$\alpha_{1}$ and $\alpha_{\mathrm{t}}\left(^{\circ}\right)$ & 120,20 \\
$Z_{m}$ & 0.1 \\
\hline$S e a l$ and bearing & \\
$C_{\text {seal }}(\mathrm{m})$ & $0.1 \times 10^{-3}$ \\
$R_{\text {seal }}(\mathrm{m})$ & $50 \times 10^{-3}$ \\
$L_{\text {seal }}(\mathrm{m})$ & $50 \times 10-3$ \\
$m_{b 1}$ and $m_{b 2}(\mathrm{~kg})$ & 2.329 \\
$m_{b 3}$ and $m_{b 4}(\mathrm{~kg})$ & 1.348 \\
\hline$S h a f t$ and rotor & \\
$k_{t 1}$ and $k_{t 2}(\mathrm{~N} / \mathrm{m})$ & $1.54 \times 105$ \\
$k_{s 1}, k_{s 2}, k_{s 3}$, and $k_{\underline{s} 4}(\mathrm{~N} / \mathrm{m})$ & $4 \times 105$ \\
$k_{s 5}(\mathrm{~N} / \mathrm{m})$ & $2.6 \times 105$ \\
$\zeta_{t}$ and $\zeta_{s}$ & 0.07 \\
$m_{r}(\mathrm{~kg})$ & 2.7 \\
$I_{r}\left(\mathrm{~kg} \cdot \mathrm{m}^{2}\right)$ & $3.72 \times 10^{-3}$ \\
\hline
\end{tabular}

dynamics of the GRSB system is analyzed by the spectrumdomain diagram, time-domain responses, bifurcation theory, and Poincare mapping.

3.1. Frequency-Domain Analysis of the GRSB System. The spectrum waterfall diagrams of the driving and driven gear in $x$ and $y$ directions are shown in Figures 8 and 9, in which the transmission ratio $i$ of the gear pair system is used as the variable parameter. Due to the coupled motion between the driving gear and the driven gear during the operation of the system, the frequency-domain responses of the system consist of the driving shaft rotational frequency $f_{p}\left(60 / n_{1}\right)$ component, the driven shaft rotational frequency $f_{g}\left(60 / n_{2}\right)$ component, the meshing frequency $f_{m}\left(z_{1} \times 60 / n_{1}\right)$ component, and the multiplicative frequency $2 f_{m}$ component, while the amplitude of the multiplicative frequency $2 f_{m}$ component is much lower than others.

In Figure 8(a), the frequency components of the driving gear in $x$ direction are mainly composed of $f_{p}$ and $f_{m}$, which means the characteristics and the motion state of the driving shaft and the meshing parameters (such as time-varying meshing stiffness/damping and internal excitation error) have a major influence on the vibration responses of the driving gear in $x$ direction. With the transmission ratio increases, the amplitude of $f_{p}$ component decreases first and then increases, reaching its minimum value at the transmission ratio of 2 . Besides, as shown in the detail, the amplitude diagram of $f_{g}$ component and the rotation of the driven gear affect the vibration responses of the driving gear, especially when the ratio is relatively small, and the amplitude of $f_{g}$ component decreases with the ratio increase. Figure 8 (b) shows the frequency-domain responses of the 


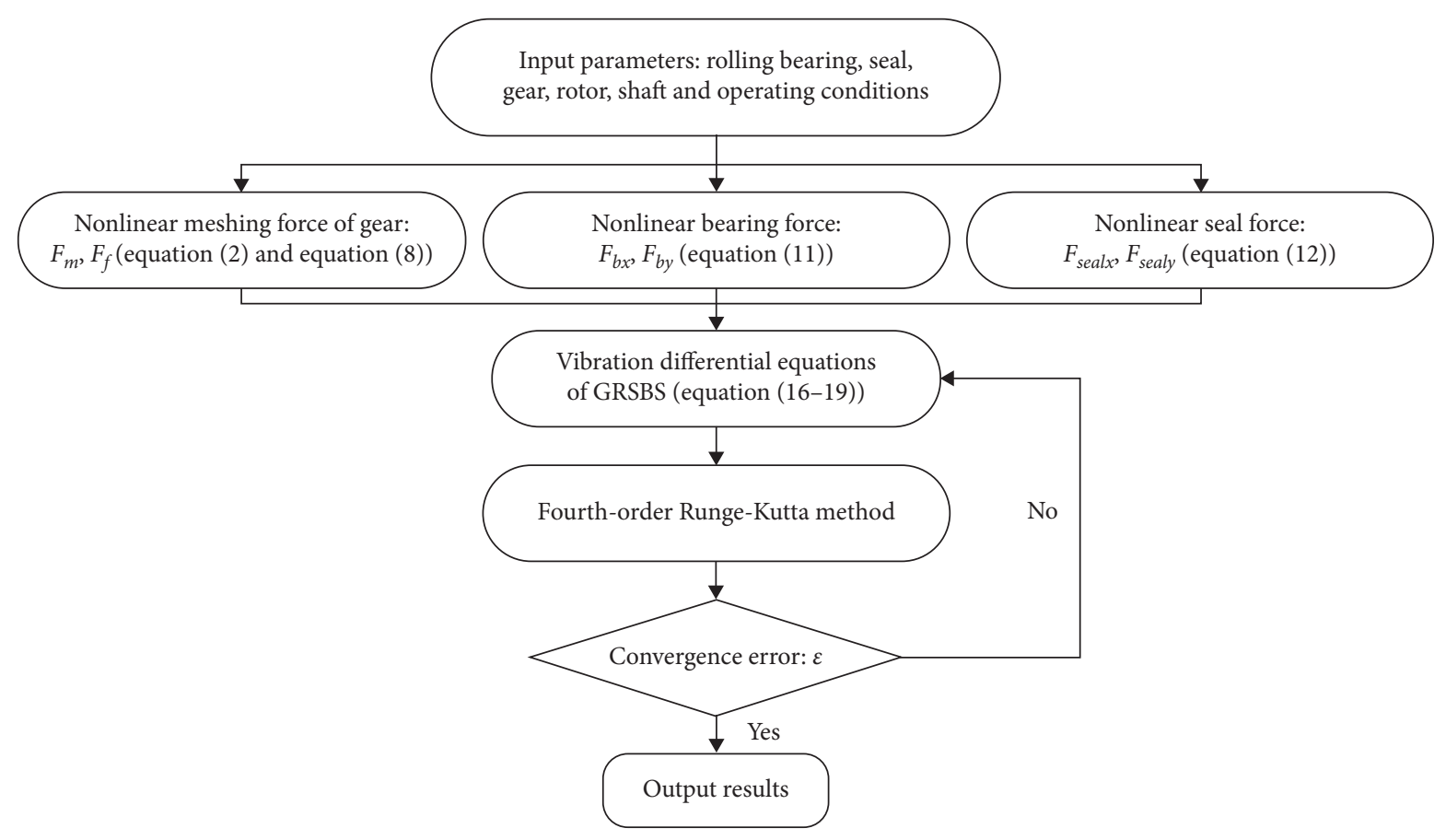

FIGURE 5: Block diagram of the nonlinear global calculation program.

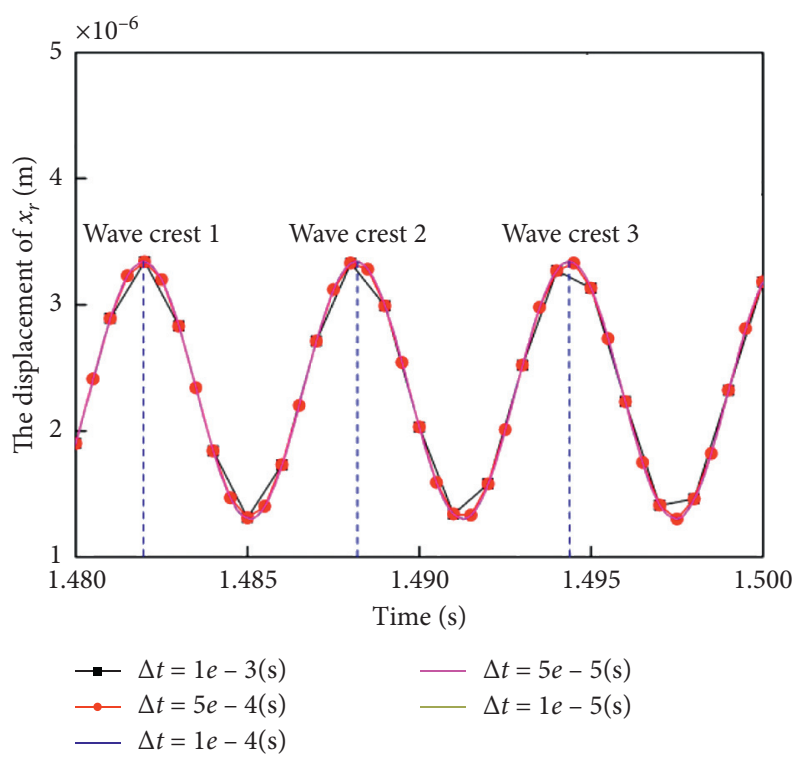

Figure 6: Time-domain diagram of $x_{r}$ with different calculation steps.

driving gear in $y$ directions at different transmission ratios. Comparing Figure 8(b) with Figure 8(a), it can be obtained that the amplitude of $f_{m}$ component of the driving gear in $y$ direction is much higher than that in $x$ direction under the same transmission ratio, which means that the vibration responses of driving gear in $y$ direction are more sensitive with the change of the meshing factors.

Figures 9(a) and 9(b) are the frequency-domain responses of the driven gear, respectively, in $x$ direction and $y$ direction. As shown in the figures, there exist the $f_{p}$ component, the $f_{g}$ component, the $f_{m}$ component, and the $2 f_{m}$ component in the

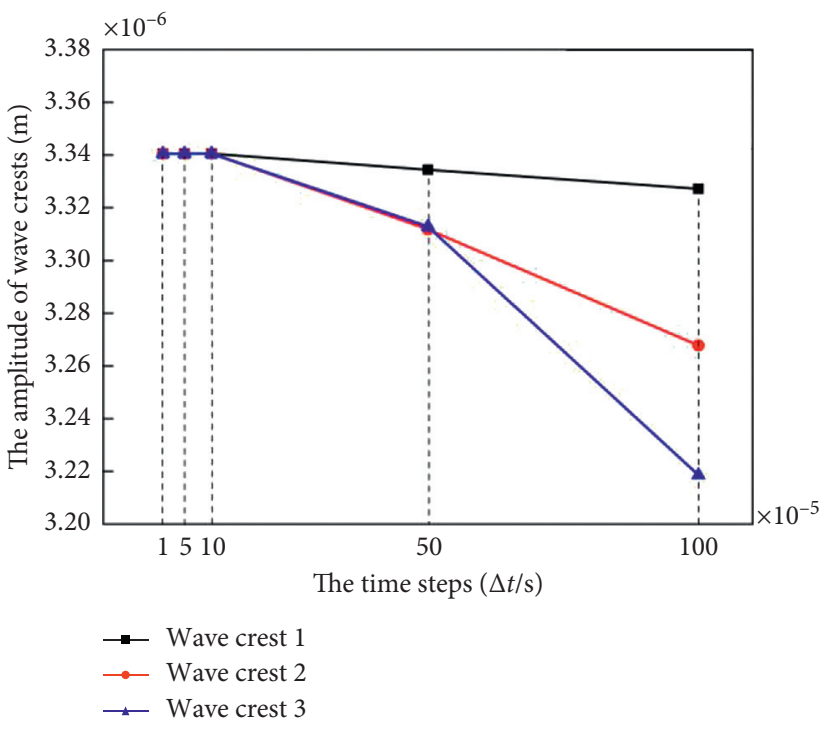

FIgURE 7: The amplitude of wave crests with different calculation steps.

frequency-domain responses. Quite different from the effects of the $f_{p}$ component on the vibration responses of the driving gear shown in Figure 8(a), $f_{g}$ component basically remains the same with the transmission ratio increase. Besides, as indicated in Figure 9(b), the amplitude of the $f_{p}$ component is higher than that of $f_{g}$, which means that the characteristics and the motion state of the driving shaft have much more impacts than those of the driven shaft on the vibration responses of the driven gear in $y$ direction.

Figures 8 and 9 illustrate that the meshing frequency $f_{m}$ component has a great influence on the vibration responses of 


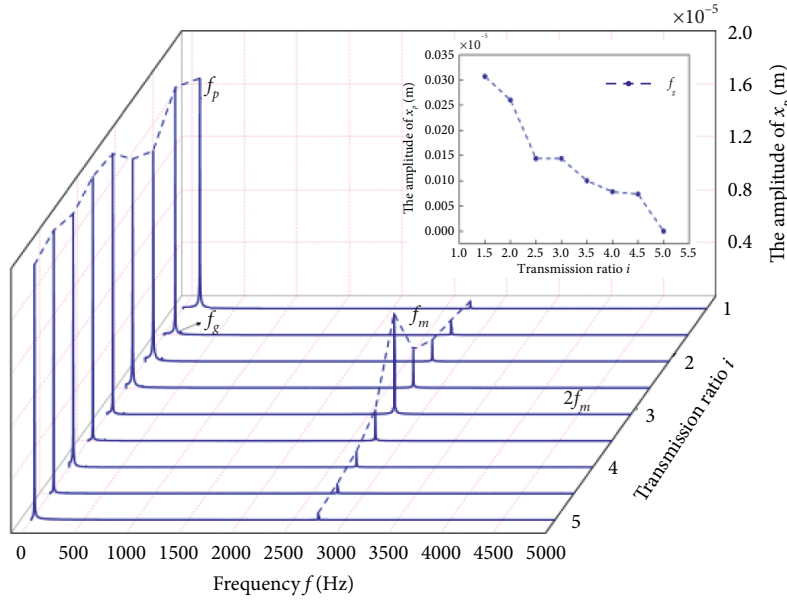

(a)

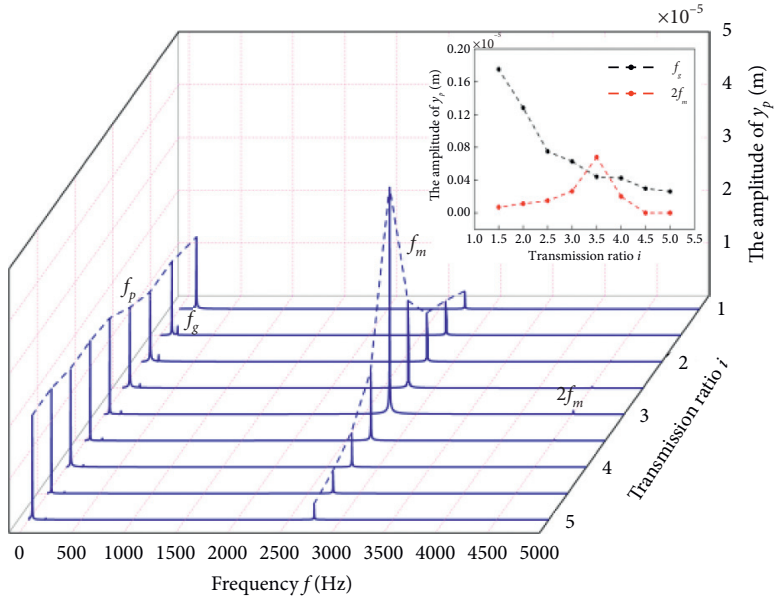

(b)

FIGURE 8: The spectrum waterfall diagram of the driving gear: (a) in $x$ direction and (b) in $y$ direction.

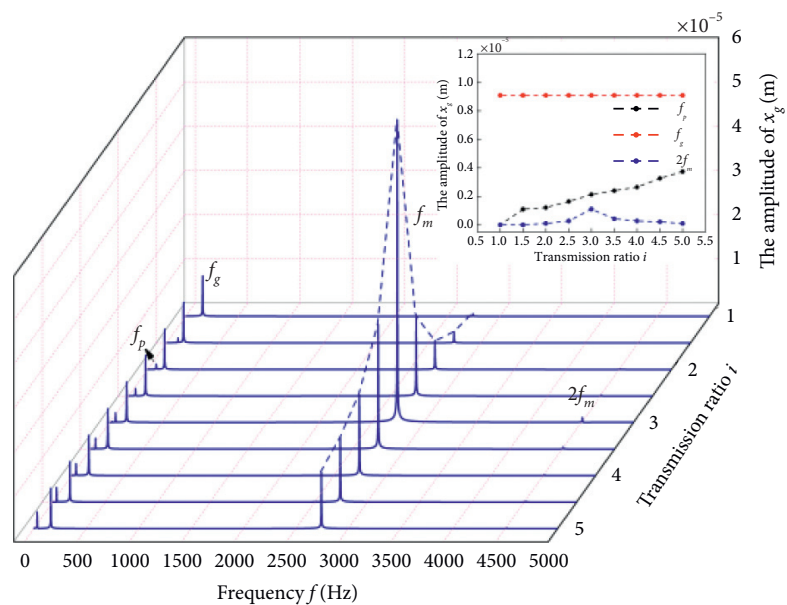

(a)

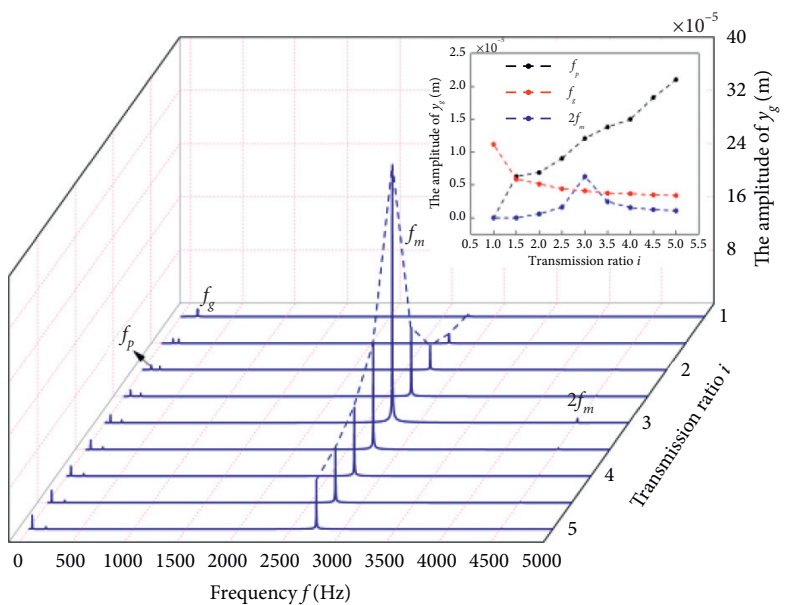

(b)

FIgURE 9: The spectrum waterfall diagram of the driven gear: (a) in $x$ direction and (b) in $y$ direction.

both the two gears. And compared with the driving gear, in both directions, the vibration responses of the driven gear are more sensitive with the change of the meshing factors. Moreover, the amplitude of the meshing frequency $f_{m}$ component increases first and then decreases obviously with the ratio increase.

Figure 10 presents the variation of $f_{m}$ component with the ratio ranging from 2.5 to 3.5 , and the variation shows that the amplitude of $f_{m}$ component reaches the maximum value under the ratio of 3 in all the four responses. Besides, as the value of $f_{m}$ is more than 17 times bigger than the other frequencies like $f_{p}$ and $f_{g}, f_{m}$ component acts as the most dominant high-frequency impact load and noise source. As shown in the figure, the amplitude of $f_{m}$ component of the driven gear in $y$ direction is 5 times higher than the amplitudes of the other components, which illustrates that the impacts and the noises of the system mainly come from the driven gear motion in $y$ direction.
3.2. Analysis of the Bifurcation Characteristics of the GRSB System. Figures 11 and 12 show the bifurcation of the gear pair in $x$ and $y$ directions with the transmission ratio as the bifurcation parameter. Figures 11(a) and 12(a) indicate that an obvious jump phenomenon appears in $x$ direction when the transmission ratio is 1.25 . As the transmission ratio increases, a bifurcation phenomenon occurs at the ratio of 4 at the driving gear. In Figure 11, the amplitude responses of the driving gear with different ratio and the distance between the highest amplitude and the lowest amplitude are almost the same, which means that there is no obvious change in fluctuation amplitude of the driving gear responses under different transmission ratio.

Figure 12 is the bifurcation diagram of the driven gear, with the increasing transmission ratio; the amplitude of the driven gear system does not change significantly until the transmission ratio is 3 . When $i$ is bigger than 3 but smaller 


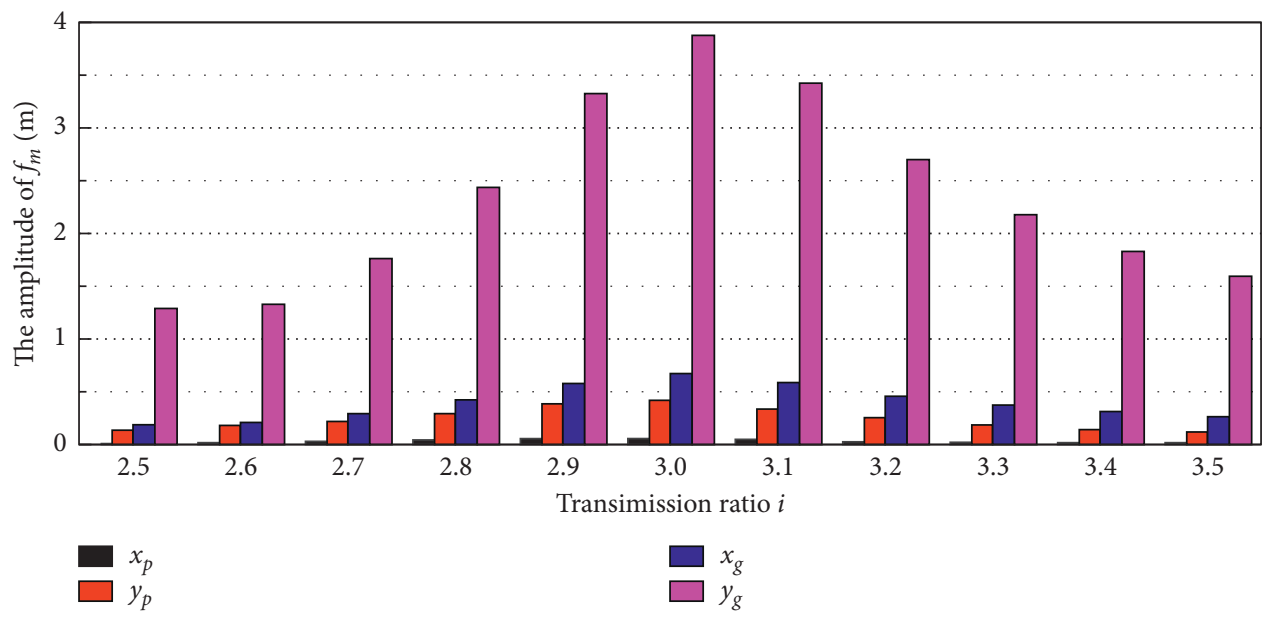

FIgURE 10: The amplitude of the meshing frequency $f_{m}$ with the change of transmission ratio.

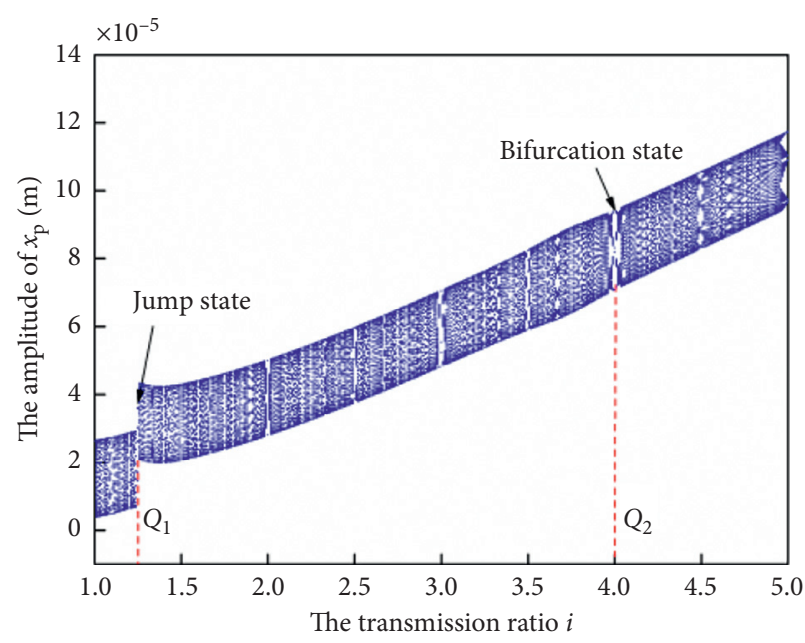

(a)

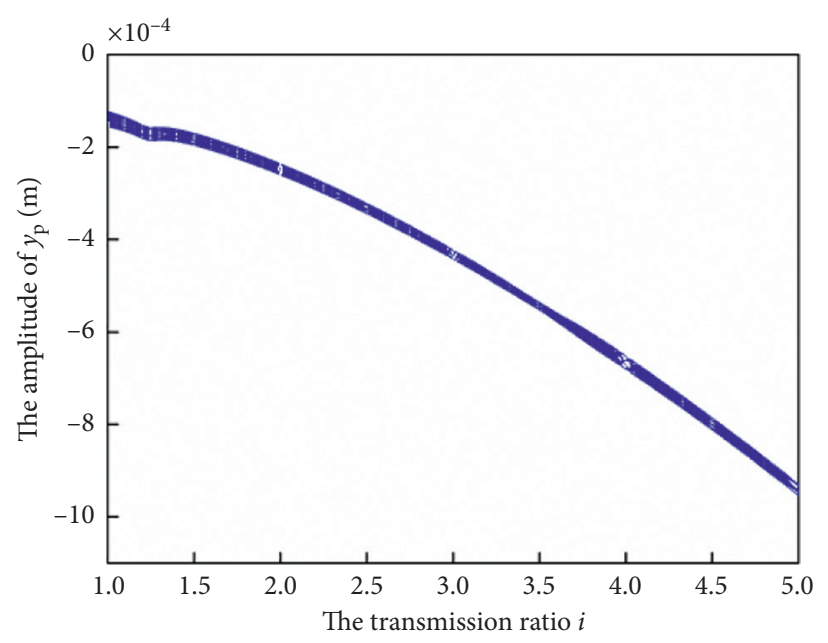

(b)

FIgURE 11: Bifurcation diagram of the driving gear: (a) in $x$ direction and (b) in $y$ direction.

than 4.5 , the fluctuation amplitude of the driven gear changed and a "lung-like" region is formed on the bifurcation diagram. In order to optimize the design of the rotor system of the high-speed centrifugal pump, the nonlinear dynamic characteristics of the driving gear and the driven gear under different motion states are compared below, including the jump states (represented by $Q_{1}$ ) and the bifurcation states (represented by $Q_{2}$ ).

\subsection{Influence of the Jump Phenomenon on the Vibration} Responses of the GRSB System. Figures 13 and 14 compare the dynamic vibration responses of the driving and driven gear in $x$ and $y$ direction under the condition that the transmission ratio is 1.25 , where an obvious jump phenomenon appeared as shown in Figure 11.

Figure 13(a) shows that the time-domain responses of $x_{p}$ have no obvious periodicity; Figure 13(b)) shows that the driving shaft rotation frequency $\left(f_{p}\right)$ component and the meshing frequency $\left(f_{m}\right)$ component are the main frequency components in the responses. Notice that there exist multiple combinations of frequencies within the frequency band lower than the driven shaft rotation frequency $\left(f_{g}\right)$ component, which is a challenge to the stability of the system. Figure 13(c) shows that the Poincare scatter is concentrated in five small separate areas. Combining Figure 13(a)) with Figure 13(c), it can be concluded that the motion of the driving gear in $x$ direction is chaotic.

Figure 14 illustrated the vibration responses of the driving gear in $y$ direction. It is obvious from the vibration responses curve that the vibration of the driving gear in the $y$ direction is more complex than that in $x$ direction. As can be seen from Figure 14(b)), the frequency-domain responses in the $y$ direction of the driving gear present obvious components with frequencies of $0.2 f_{p}$ and $0.5 f_{g}$. Moreover, combining with the facts that the amplitude of $f_{m}$ component in $y$ direction is much larger than that in $x$ direction, the multiplicative frequency $2 f_{m}$ component appears in $y$ direction too, and it can be obtained that the impacts of the meshing are stronger on the vibration in $y$ direction. The 


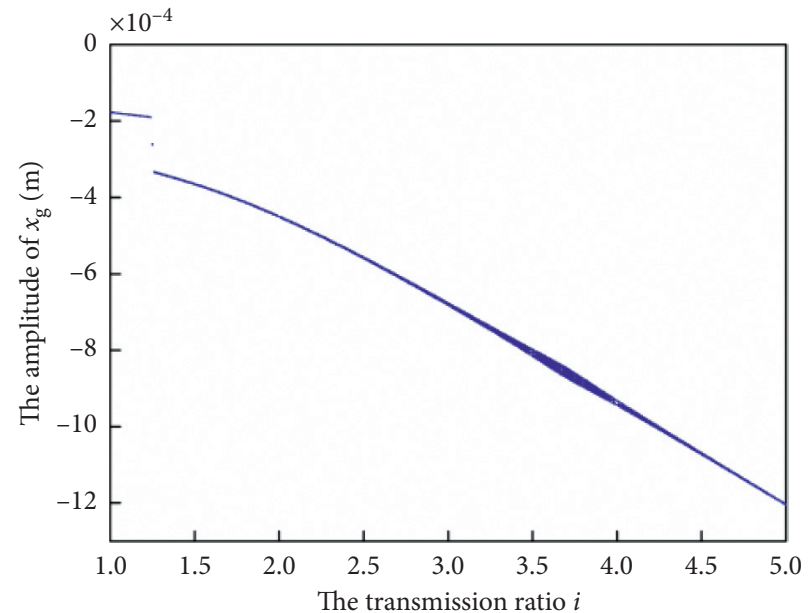

(a)

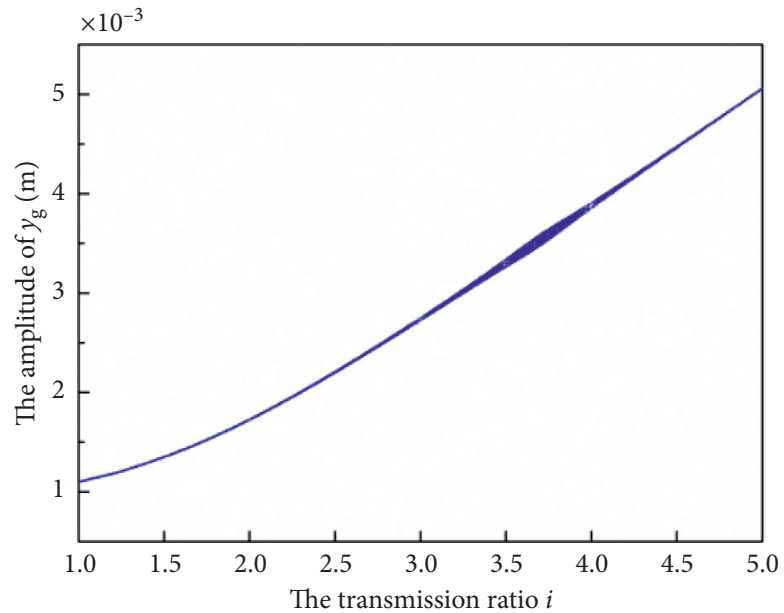

(b)

Figure 12: Bifurcation diagram of the driven gear: (a) in $x$ direction and (b) in $y$ direction.

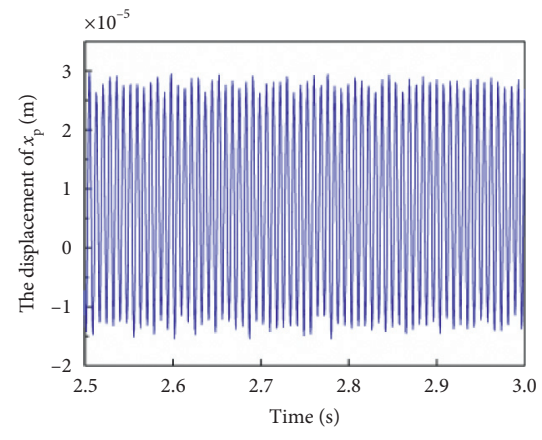

(a)

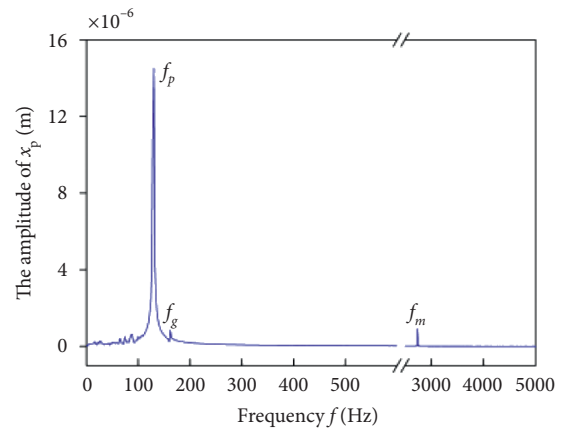

(b)

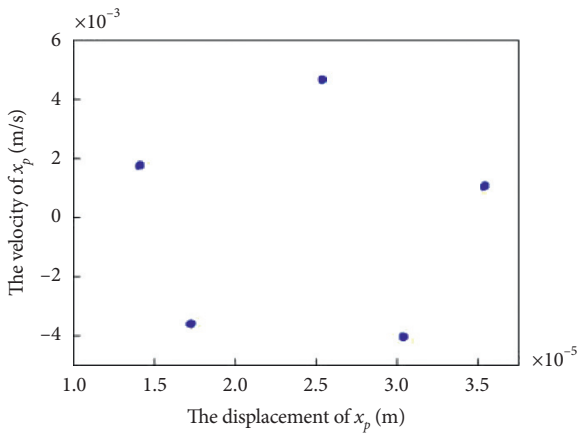

(c)

FIgURe 13: The dynamic vibration responses of $x_{p}$ at $i=1.25$. (a) Time-domain diagram. (b) Frequency-domain diagram. (c) Poincare map.

scatter of Poincare map of $y_{p}$ shows that the motion state of the driving gear in $y$ direction is chaotic, and the stability in $y$ direction is worse than that in $x$ direction.

Figures 15 and 16 are, respectively, the frequency-domain responses and Poincare mapping of the driven gear. Compared with the driving gear, the amplitude of $0.2 f_{p}$ component is particularly large, and it has become the most contributed frequency in the vibration responses. The meshing frequency $\mathrm{fm}$ has become one of the dominant frequencies of the driven gear vibration responses. And Figure 16(a) shows that the driving gear is more stable than the driven gear in $x$ direction. Comparing Figures 16(a) and 16(b), the scatter distribution in $y$ direction shows better stability than in $x$ direction, which is quite different from the distributions of the driving gear.

3.4. Influence of the Bifurcation on the Vibration Responses of the GRSB System. To further analyze the response characteristics of the GRSB system under bifurcation periodic motion state, the frequency-domain diagram and the Poincare map are used to describe the vibration responses of the gears under the transmission ratio of 4 .

Comparisons of the frequency-domain responses of the gears shown in Figure 17 and the responses of the gears shown in Figures 13 and 14 illustrate that the frequency components under bifurcation periodic motion state are simpler than that the jump state, which includes only $f_{p}$ (the driving rotational speed frequency) component, $f_{g}$ (the driven rotational speed frequency) component, $f_{m}$ (the meshing frequency) component, and $2 f_{m}$ (the multiplicative frequency) component but with no low-frequency components like $0.2 f_{p}$ and $0.5 f_{g}$. Moreover, the vibration responses of the driving gear are heavily dependent on $f_{p}$ component. In contrast, $f_{m}$ acts as the first dominating factor in the driven gear responses. The amplitude of $f_{m}$ component in the driven is significantly higher than that in the driving gear in both the two directions. And the meshing frequency $f_{m}$ 


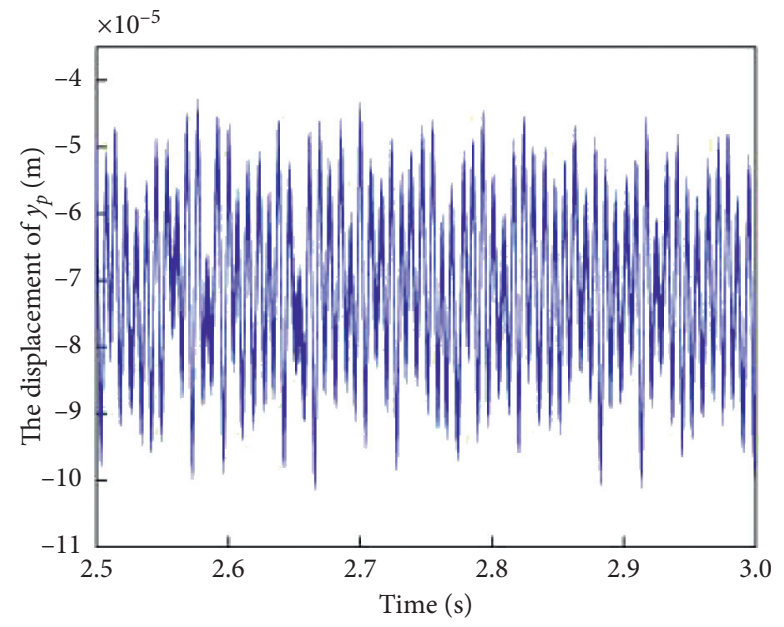

(a)

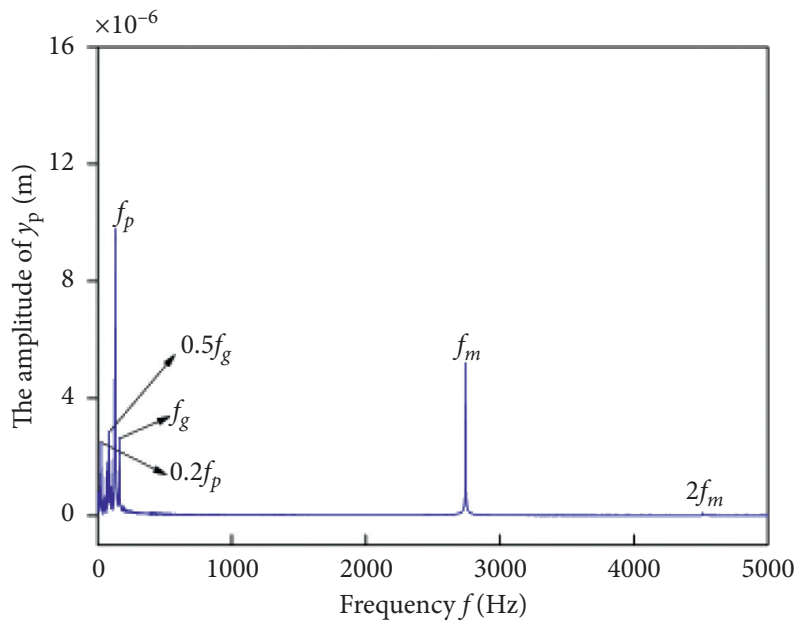

(b)

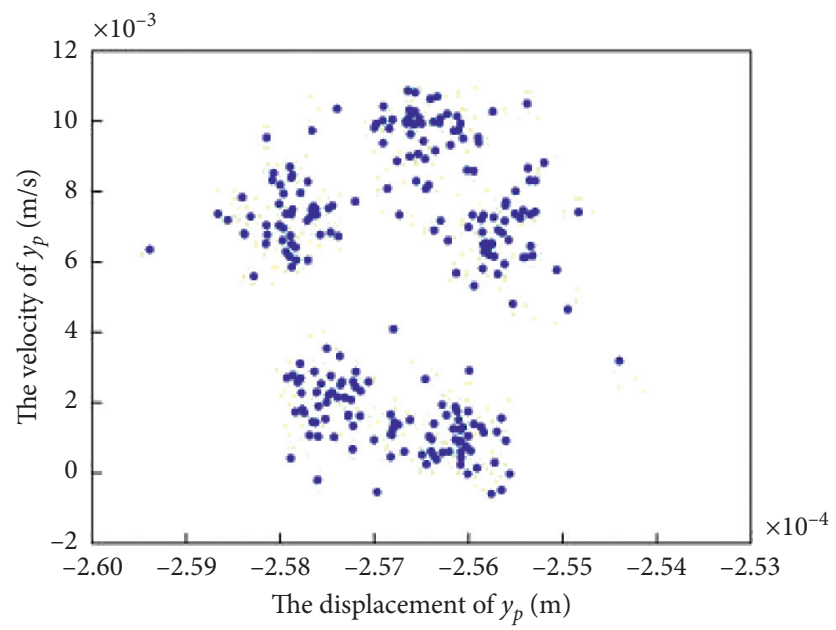

(c)

FIgURE 14: The dynamic vibration responses of $y_{p}$ at $i=1.25$. (a) Time-domain diagram. (b) Frequency-domain diagram. (c) Poincare map.

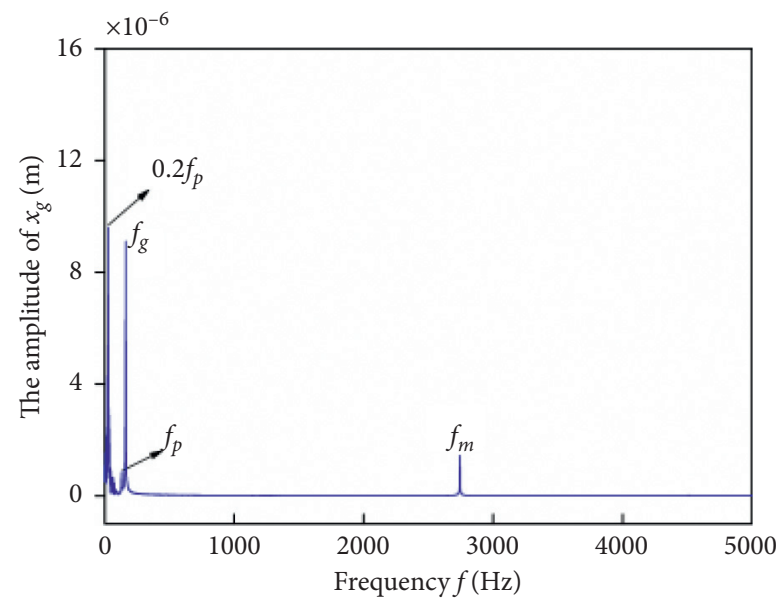

(a)

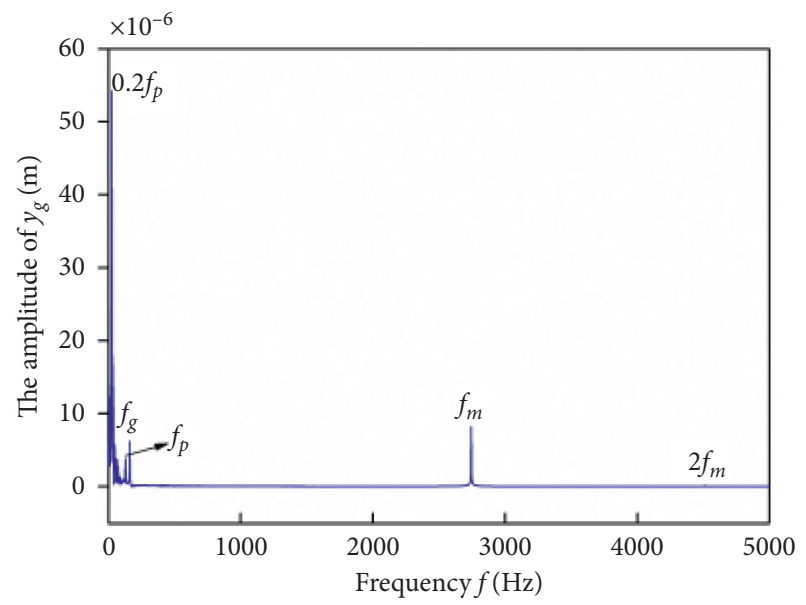

(b)

Figure 15: The frequency-domain responses of driven gear at $i=1.25$ : (a) in $x$ direction and (b) in $y$ direction. 


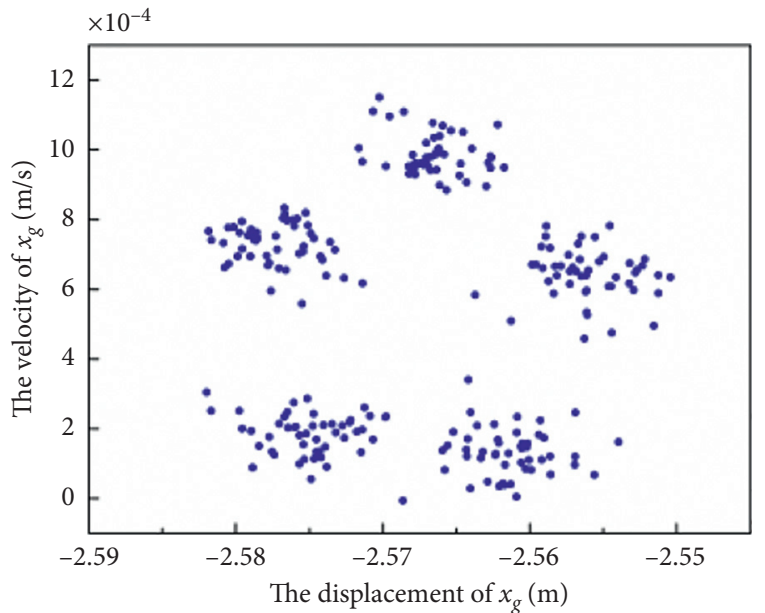

(a)

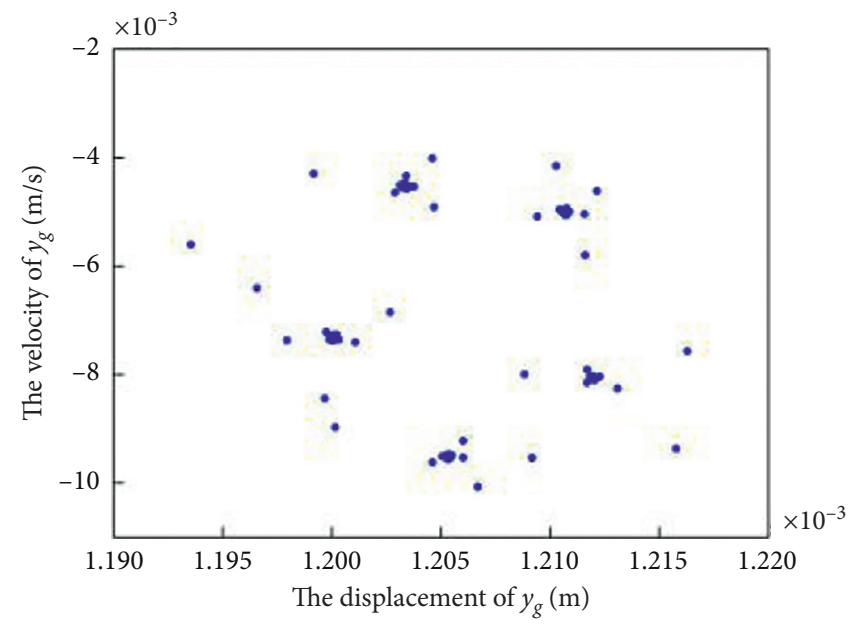

(b)

FIgURE 16: The Poincare mapping of driven gear at $i=1.25$ : (a) in $x$ direction and (b) in $y$ direction.

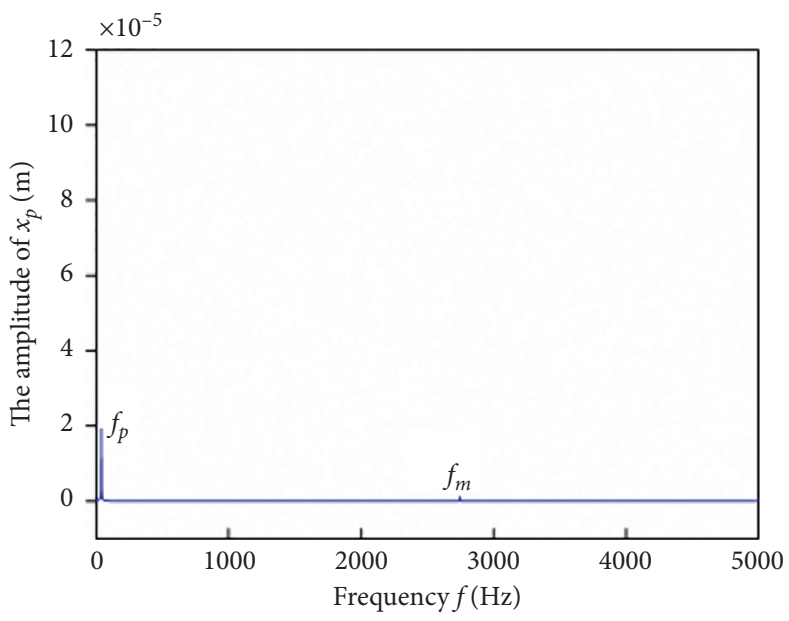

(a)

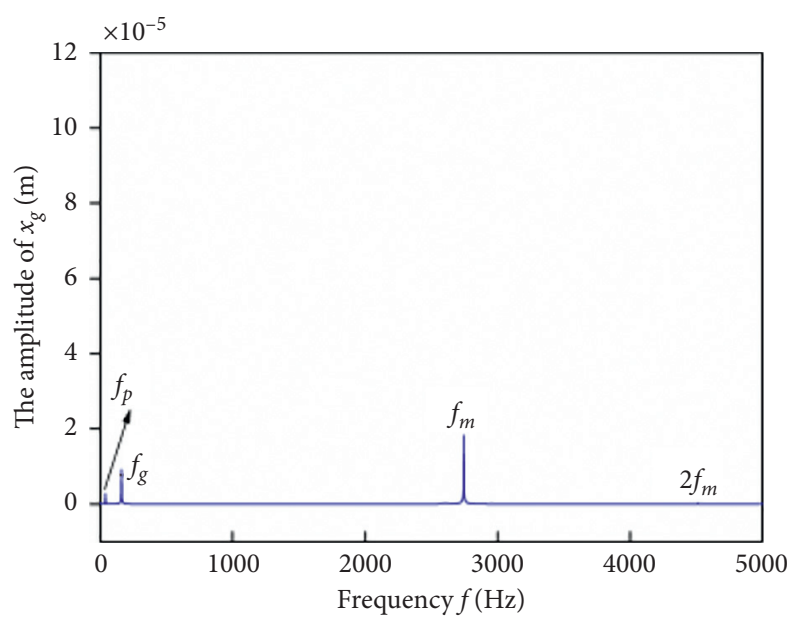

(c)

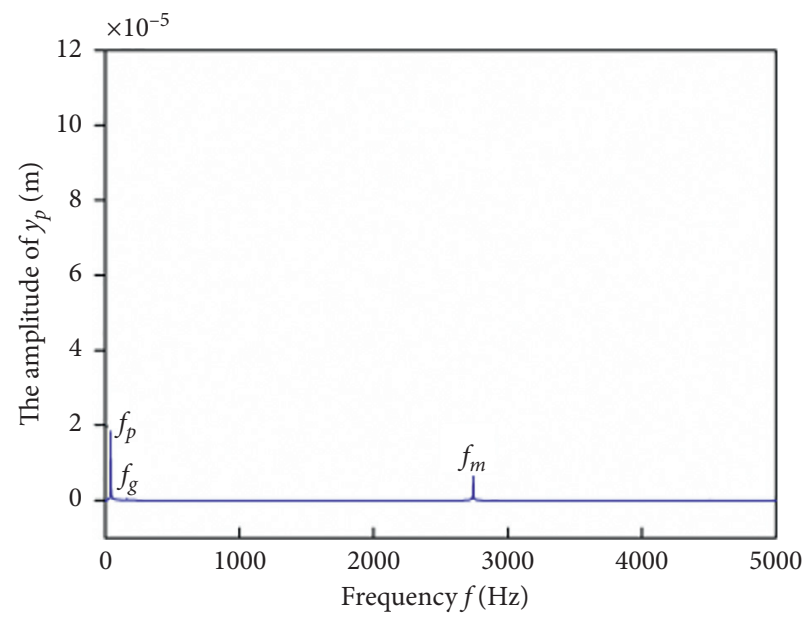

(b)

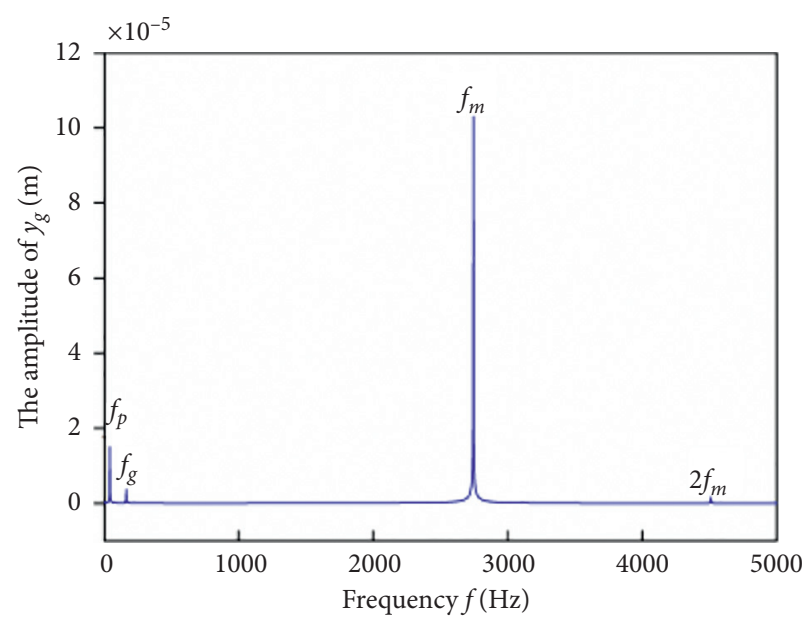

(d)

Figure 17: The frequency-domain responses at $i=4$. (a) The driving gear in $x$ direction. (b) The driving gear in $y$ direction. (c) The driven gear in $x$ direction. (d) The driven gear in $y$ direction. 


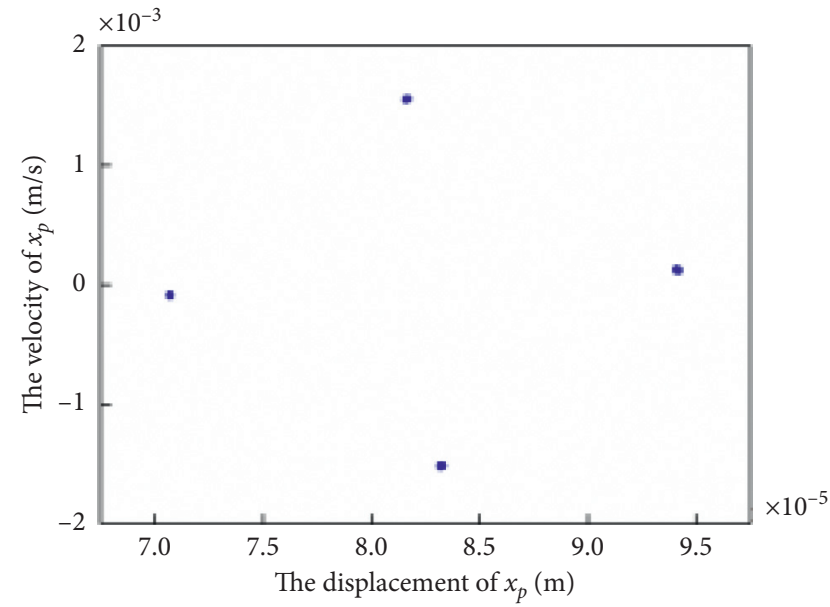

(a)

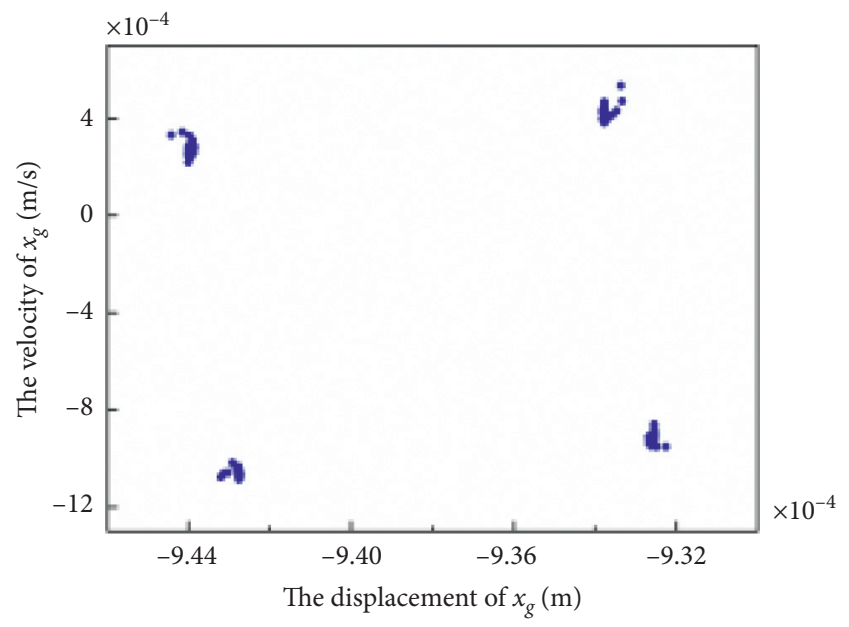

(c)

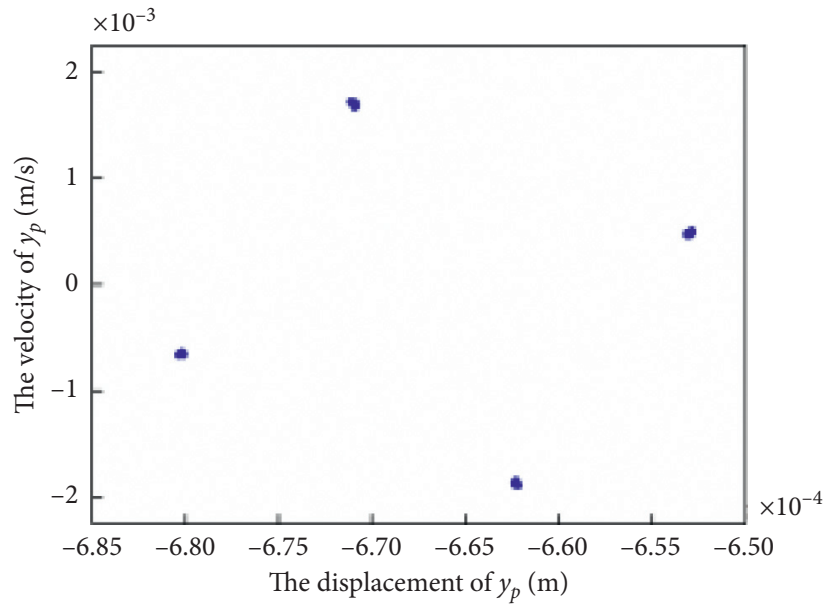

(b)

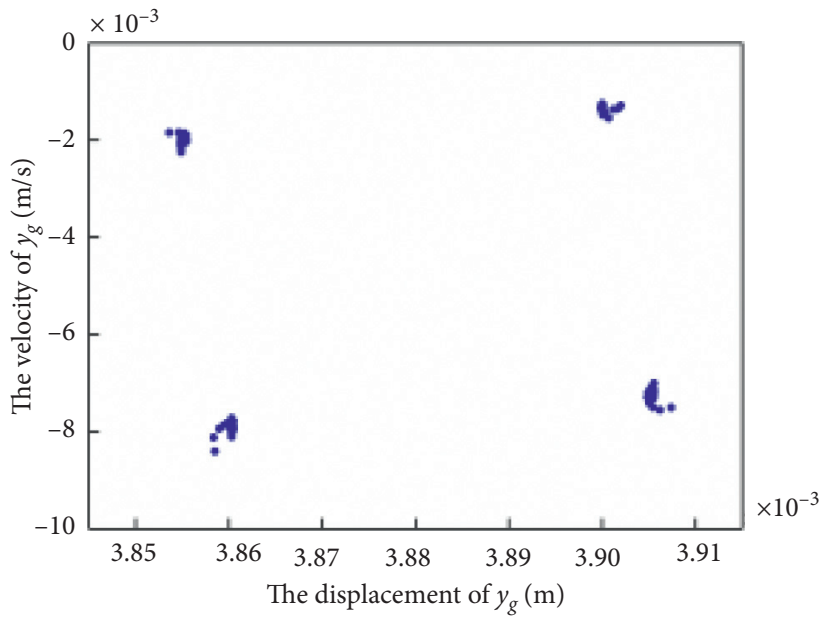

(d)

Figure 18: The Poincare mapping at $i=4$. (a) The driving gear in $x$ direction. (b) The driving gear in $y$ direction. (c) The driven gear in $x$ direction. (d) The driven gear in $y$ direction.

component has more contribution of the gear responses in $y$ direction than in $x$ direction. Figure 18 illustrates that the gears under the bifurcation state with a transmission ratio of 4 are far more stable than in the jump state with a transmission ratio of 1.25 .

\section{Conclusions}

In this study, a coupled lateral-torsional vibration dynamic model of a high-speed centrifugal pump rotor considering the nonlinear bearing forces, nonlinear seal forces, the tooth surface friction, mass eccentricity, backlash, and the internal error of gears is established. Some conclusions that will help the optimal design for high-speed centrifugal pumps can be drawn as follows:

(1) Due to the coupling motion of the system, the frequency-domain responses in both directions are composed of $f_{p}$ (the rotation speed of driving shaft frequency) component, $f_{g}$ (the rotation speed of driven shaft frequency) component, $f_{m}$ (the meshing frequency) component, and $2 f_{m}$ (the multiplicative frequency) component. Moreover, $f_{m}$ component has a significant influence on the vibration responses of the driving and driven gears, and the amplitude of $f_{m}$ component increases first, then decreases with the ratio increase, and reaches the maximum value under the ratio of 3 .

(2) The motion state of the gears is sensitive to the change of the transmission ratio of the GRSB system. The vibration responses of the gear system in $x$ direction will appear in the jump state when the ratio is 1.25 and bifurcation state when the ratio is 4 . Comparisons of the vibration responses and Poincare mapping show that the system is more stable in the bifurcation state than in the jump state, and the frequency composition of the responses is relatively simpler.

(3) When the system is in the jump motion state, the low frequencies of $0.2 f_{g}$ and $0.5 f_{p}$ will appear in both gears in two directions, which are also the most 
contributed factors to the vibration responses of the driven gear. Besides, the stability of the driving gear and the driven gear is opposite in the two directions; that is, the driving gear has better stability in $x$ direction, while the driven gear has better one in $y$ direction.

(4) In the design process of a high-speed centrifugal pump with a simplified GRSB system, the transmission ratio should avoid the jump-state design $(i=1.25)$ to ensure the stability of the system and avoid the maximum-amplitude-of $-f_{m}$ design $(i=3)$ to reduce the mechanical impacts and noises.

\section{Nomenclature}

\section{Subscripts}

am: Amplitude

Av: Average

$B$ : Node of rolling bearing

$D$ : Node acted by input load

$F$ : Friction of gears

$G$ : Node of driven gear

$M$ : Meshing processes

$P: \quad$ Node of driving gear

$R: \quad$ Node of rotor

seal: Node of annular seal

$X: \quad$ In $x$ direction

$Y: \quad$ In $y$ direction.

\section{Symbols}

backlash: Gear backlash

C: $\quad$ Damping

E: $\quad$ Mass eccentricity

$f: \quad$ Frequency

F: $\quad$ Force

G: $\quad$ Barycenter

$i$ : Transmission ratio

I: $\quad$ Rotational inertia

$k: \quad$ Stiffness

$k_{s}$ : $\quad$ Bending stiffness

$k_{t}$ : Torsional stiffness

$\mathrm{Nb}$ : Number of the roller

$m$ : $\quad$ Equivalent mass

M: $\quad$ Model of gear

$n_{1} / n_{2}$ : Rotational speed of driving/driven shaft

$o: \quad$ Equivalent mass point

S: $\quad$ Relative displacement

T: $\quad$ Torque

$x: \quad$ Displacement of $x$ direction

$y: \quad$ Displacement of $y$ direction

$z: \quad$ Teeth of gear

$\alpha_{1}$ : $\quad$ Angle between the center line and the axis of the gear

$\alpha_{t}$ : $\quad$ Pressure angle of gear

$\delta: \quad$ Error

$\Delta: \quad$ Deformation of roller

$\varepsilon: \quad$ Convergence error

$\zeta: \quad$ Damping ratio $\theta: \quad$ Torsional displacement

$\lambda: \quad$ Direction coefficient of the gear tooth friction

$\varphi$ : $\quad$ Angular velocity

$\eta$ : $\quad$ Transmission efficiency.

\section{Data Availability}

The numerical and experimental data used to support the findings of this study are included within the article.

\section{Conflicts of Interest}

The authors declare that there are no conflicts of interest regarding the publication of this paper.

\section{Acknowledgments}

This work was supported by the National Natural Science Foundation of China (Grant no. 51976199), the Joint Project from the National Natural Science Foundation of China (Grant no. U1608258), and the Zhejiang Province Public Welfare Technology Research Project (Grant no. LGG18E060007).

\section{References}

[1] G. Peng, X. Huang, L. Zhou, G. Zhou, and H. Zhou, "Solidliquid two-phase flow and wear analysis in a large-scale centrifugal slurry pump," Engineering Failure Analysis, vol. 114, p. 104602, 2020.

[2] W. J. Zhou, Y. C. Yang, G. K. Xing, and L. Q. Wang, "Numerical investigation of nonlinear vibration for rotor-seal system of centrifugal pump," IO Conference Series: Materials Science and Engineering, vol. 52, Article ID 3320061, 2013.

[3] Y. Yang, L. Zhou, J. Hang, D. Du, W. Shi, and Z. He, "Energy characteristics and optimal design of diffuser meridian in an electrical submersible pump," Renewable Energy, vol. 196, Article ID 107653, 2020.

[4] Y. Yang, L. Zhou, W. Shi, Z. He, Y. Han, and Y. Xiao, "Interstage difference of pressure pulsation in a three-stage electrical submersible pump," Journal of Petroleum Science and Engineering, vol. 196, 2020.

[5] S. H. Zhou, G. Q. Song, M. N. Sun, and Z. H. Ren, "Nonlinear dynamic analysis for high speed gear-rotor-bearing system of the large scale wind Turbine," Journal of Vibration Engineering, vol. 8, no. 17, pp. 4560-4574, 2015.

[6] H. R. El-Sayed, "Stiffness of deep groove ball bearings," Wear, vol. 63, pp. 89-94, 1980.

[7] S. P. Harsha, K. Sandeep, and R. Prakash, "Effects of preload and number of balls on nonlinear dynamic behavior of ball bearing system," International Journal of Nonlinear Science Numerical Simulation, vol. 4, no. 3, pp. 265-278, 2003.

[8] S. P. Harsha, K. Sandeep, and R. Prakash, "Nonlinear dynamic behaviors of rolling element bearings due to surface waviness," Journal of Sound Vibration, vol. 272, pp. 557-580, 2004.

[9] S. P. Harsha and P. K. Kankar, "Stability analysis of a rotor bearing system due to surface waviness and number of balls," International Journal of Mechanical Sciences, vol. 46, no. 7, pp. 1057-1081, 2004.

[10] S. P. Harsha, "Nonlinear dynamic analysis of a high-speed rotor supported by rolling element bearings," Journal of Sound and Vibration, vol. 290, pp. 65-100, 2006. 
[11] P. K. Kankar, S. C. Sharma, and S. P. Harsha, "Nonlinear vibration signature analysis of a high speed rotor bearing system due to race imperfection," Journal of Computational and Nonlinear Dynamics, vol. 7, 2012.

[12] W. Z. Wang, S. Zhang, Z. Zhao, and S. Ai, "Effect of the in homogeneity in races on the dynamic behavior of rolling bearing," Journal of Vibration and Acoustics Transactions of the ASME, vol. 6, no. 137, 2015.

[13] R. Yang, L. Hou, Y. Jin, Y. Chen, and Z. Zhang, "The varying compliance resonance in a ball bearing rotor system Affected by different ball numbers and rotor eccentricities," Journal of Tribology, vol. 140, 2018.

[14] A. Muszynska and D. E. Bently, "Improvements in lightly loaded rotor/bearing and rotor/seal models," Journal of Vibration, Acoustics, Stress, and Reliability in Design, vol. 110, no. 2, pp. 129-136, 1988.

[15] J. E. Leung, "Hopf bifurcation analysis of a rotor/seal system," Journal of Sound and Vibration, vol. 252, no. 5, pp. 817-833, 2002.

[16] M. Cheng, G. Meng, and J. P. Jing, "Nonlinear dynamics of a rotor-bearing-seal system," Journal of Sound and Vibration, vol. 76, pp. 215-227, 2006.

[17] W. Li and Y. Yi, "Nonlinear dynamic analysis of a rotor bearing seal system," Journal of Zhejiang University-SCIENCE A (Applied Physics \& Engineering), vol. 12, no. 1, pp. 46-55, 2010.

[18] S. Peng, A. Arai, T. Fukuchi, T. Katayama, and O. Matsushita, "Rotor stability evaluation for high pressure muliti-stage pump by excitation diagnosis method," International Gas Turbine and Aeroengine Congress and Exposition, vol. 69191, 2017.

[19] E. Zhang, Y. Jiao, and Z. Chen, "Dynamic behavior analysis of a rotor system based on a nonlinear labyrinth-seal forces model," Journal of Computational and Nonlinear Dynamics, vol. 13, 2018.

[20] W. J. Zhou, X. S. Wei, L. L. Zhai, and L. Q. Wang, "Nonlinear characteristics and stability optimization of rotor-seal-bearing system," Journal of Vibroengineering, vol. 16, pp. 817-832, 2014.

[21] W. J. Zhou, X. S. Wei, and L. Q. Wang, "Numerical research on dynamic lateral vibration of a pump turbine's shaft system," Journal of Engineering Research, vol. 3, no. 4, pp. 127$148,2015$.

[22] L. Q. Wang and W. J. Zhou, "A coupling vibration model of multi-stage pump rotor system based on FEM," Mechanika, vol. 22, no. 1, pp. 31-37, 2016.

[23] W. J. Zhou, N. Qiu, N. Zhang, Z. Lai, and B. Gao, "Steady state characteristics of centrifugal pump rotor system with weak nonlinear stiffness," Transactions of Famena Xlii, vol. 3, pp. 87-102, 2018.

[24] L. Bai, L. Zhou, X. Jiang, Q. Pang, and D. Ye, "Vibration in a multistage centrifugal pump under varied conditions," Shock and Vibration, vol. 2019, Article ID 2057031, 9 pages, 2019.

[25] A. Kaharman and R. Singh, "Nonlinear dynamics of a spur gear pair," Journal of Sound and Vibration, vol. 142, pp. 49-75, 1990.

[26] A. Kaharman, "Nonlinear dynamics of a geared rotor-bearing system with multiple clearances," Journal of Sound and Vibration, vol. 144, pp. 4469-4506, 1991.

[27] J. S. Rao, T. N. Shuai, and J. R. Chang, "Theoretical analysis of lateral responses due to torsional excitation of geared rotors," Mechanism and Machine Theory, vol. 33, pp. 761-783, 1998.
[28] S. N. Stephanos, "On geared rotor dynamic systems with oil journal bearings," Journal of Sound and Vibration, vol. 243, no. 4, pp. 721-745, 2001.

[29] L. Vedmar and A. Anette, "A method to determine dynamic loads on spur gear teeth and on bearings," Journal of Sound and Vibration, vol. 265, no. 5, pp. 1065-1084, 2003.

[30] A. S. Lee and J. W. Ha, "Prediction of maximum unbalance responses of a gear-coupled two-shaft rotor-bearing system," Journal of Sound and Vibration, vol. 282, pp. 507-523, 2005.

[31] A. S. Anoshirvan, "Global bifurcation and chaos analysis in nonlinear vibration of spur gear systems," Nonlinear Dynamics, vol. 75, no. 4, pp. 783-806, 2014.

[32] C. F. Li, S. H. Zhou, J. X. Xie, and B. C. Wen, "Coupled lateral torsional axial vibrations of a helical gear-rotor-bearing system," Acta Mechanica Sinica, vol. 30, no. 5, pp. 746-761, 2014.

[33] M. Habibollah, S. Farhad, and M. Moslem, "Nonlinear vibration of the bevel gear with teeth profile modification," Nonlinear Dynamic, vol. 83, pp. 1875-1884, 2016.

[34] M. Jerzy, "Modelling of the gear backlash," Nonlinear Dynamics, vol. 97, no. 1, pp. 355-368, 2019.

[35] S. Tang, S. Yuan, and Y. Zhu, "Convolutional neural network in intelligent fault diagnosis toward rotatory machinery," IEEE Access, vol. 8, no. 1, pp. 86510-86519, 2020.

[36] R. Kasuba and J. W. Evans, "An extended model for determining dynamic loads in spur gearing," Journal of Mechanical Design, vol. 103, no. 3, pp. 398-409, 1981.

[37] X. P. Li, J. C. Xu, Z. M. Yang, R. Z. Chen, and H. X. Yang, "The influence of tooth surface wear on dynamic characteristics of gear-bearing system based on fractal theory," Journal of Computational and Nonlinear Dynamics, vol. 15, 2020.

[38] D. W. Childs, "Dynamic analysis of turbulent annular seals based on Hirs' lubrication equation," Journal of Lubrication Technology, vol. 105, no. 3, pp. 429-436, 1983. 